\title{
Experimental observation and investigation of the prewave zone effect in optical diffraction radiation
}

\author{
P. Karataev, ${ }^{1, *}$ S. Araki, ${ }^{2}$ A. Aryshev, ${ }^{1}$ G. Naumenko, ${ }^{3}$ A. Potylitsyn, ${ }^{3}$ N. Terunuma,${ }^{2}$ and J. Urakawa ${ }^{2}$ \\ ${ }^{1}$ John Adams Institute at Royal Holloway, University of London, Egham, Surrey TW20 OEX, United Kingdom \\ ${ }^{2}$ KEK: High Energy Accelerator Research Organization, 1-1 Oho, 305-0801 Tsukuba, Ibaraki, Japan \\ ${ }^{3}$ Tomsk Polytechnic University, 634050, prospekt Lenina 2a, Tomsk, Russia
}

(Received 31 July 2007; published 10 March 2008)

\begin{abstract}
Transition radiation (TR) and diffraction radiation (DR) has widely been used for both electron beam diagnostics and generation of intense radiation beams in the millimeter and the submillimeter wavelength range. Recently, it was theoretically predicted that TR and DR properties change either at extremely high energies of electrons or at long radiation wavelengths. This phenomenon was called a prewave zone effect. We have performed the first observation and detailed investigation of the prewave zone effect in optical diffraction radiation at $1.28 \mathrm{GeV}$ electron beam at the KEK-Accelerator Test Facility (KEK-ATF). The beam energy at KEK-ATF is definitely not the highest one achieved in the world. Since we could easily observe the effect, at higher energies it might cause serious problems. We developed and applied a method for prewave zone suppression valid for optical wavelengths. Furthermore, a method for prewave zone suppression applicable for longer radiation wavelengths is discussed.
\end{abstract}

DOI: 10.1103/PhysRevSTAB.11.032804

PACS numbers: $41.60 .-\mathrm{m}, 41.75 .-\mathrm{i}$

\section{INTRODUCTION}

Up-to-date transition radiation (TR) and diffraction radiation (DR) appearing when a charged particle moves through or passes nearby a medium has widely been used for both electron beam diagnostics [1-6] and generation of intense radiation beams in the millimeter and the submillimeter wavelength range [7-10]. In spite of the fact that TR and DR have been intensively theoretically and experimentally investigated for over 50 years, recently it was theoretically predicted [11] that TR and DR spatialspectral properties change either at extremely high energies of electrons or at long wavelengths of the emitted radiation. This phenomenon was called the prewave zone effect (PWZ). Afterwards, many scientists have performed rigorous theoretical investigation of the $\mathrm{PWZ}$ effect in TR phenomenon [11-16].

A field of emitted radiation is usually considered in two zones: near-field zone and far-field zone. The near-field zone is considered at the distance from the source comparable to its transverse dimensions. This definition is frequently used for laser radiation. In this case the theoretical models have the most general as well as the most complicated representations. The far-field zone is an approximation, which might be used at comparatively large distances from the source. In laser physics the far-field zone is frequently considered at the distance from the source much larger than its transverse dimensions. In this case the theoretical consideration is significantly simplified, e.g., the radiation field in the far-field zone can be consid-

\footnotetext{
*Corresponding author. karataev@pp.rhul.ac.uk
}

ered as a Fourier transform of the radiation source, i.e., Fraunhofer diffraction.

However, this determination of the far-field zone is not valid for radiation emitted by a source induced by a relativistic charged particle. The radiation emitted by the source has an intrinsic angular divergence of order of $\sim \gamma^{-1}$, where $\gamma$ is the charged particle Lorentz factor. The effective radiation source size is defined as $\gamma \lambda / 2 \pi$ (see, for instance, [17]). In order to obey the far-field approximation, the radiation source size should be much smaller than the radiation spatial distribution at the observation plane, i.e.

$$
\frac{\gamma \lambda}{2 \pi} \ll \frac{L}{\gamma}
$$

Here $\lambda$ is the radiation wavelength and $L$ is the distance from the source to the observation plane. Using Eq. (1), one can formulate the PWZ condition:

$$
\frac{\gamma \lambda}{2 \pi} \ll L \leq \frac{\gamma^{2} \lambda}{2 \pi} .
$$

From Eq. (2), the PWZ is the distance from the source much larger than its transverse dimensions, but not large enough to apply the far-field approximation (i.e. PWZ is in between the near-field and far-field zones). It is clear that the PWZ effect becomes severe at extremely high energies. For example, for SLAC final focus test beam (FFTB) energy of $30 \mathrm{GeV}$ [1] $(\gamma=60000)$ and an optical wavelength $\lambda=500 \mathrm{~nm}$, the far-field approximation should be used at the distance of a few kilometers, which is very difficult to achieve in practice. Furthermore, the PWZ effect can be significant at moderate relativistic energies, if the radiation wavelength of interest is too long. For 
example, for a $50 \mathrm{MeV}$ electron beam and $1 \mathrm{~mm}$ radiation wavelength, according to Eq. (1) the far-field approximation can be used at the distance $L>10 \mathrm{~m}$.

The first observation of the PWZ effect in optical transition radiation (OTR) was presented in [18]. The authors presented some broadening of the OTR spatial distribution for two different wavelengths. The experimental results were in good agreement with the theoretical expectations. However, there was a weak doubt about if they had really observed the PWZ effect. Our theoretical considerations made us believe that a certain target deformation might lead to a similar behavior of the OTR spatial distribution. For example, in [19] it was clearly shown that TR or DR in the PWZ at the distance, $L$, from the detector have similar characteristics as the radiation in the far-field zone (targetto-detector distance tends to infinity) from a concave (or convex) target with curvature radius, $R$.

Therefore, we needed an alternative confirmation about the PWZ existence. Furthermore, the PWZ effect in optical diffraction radiation (ODR) was expected as well. The theory predicted some specific peculiarities of the ODR spatial characteristics, which are absent in the far-field zone. A detailed investigation of ODR properties could give a lot of information about the PWZ effect and problems related to it.

We have performed the first observation and detailed investigation of the PWZ effect in ODR at $1.28 \mathrm{GeV}$ electron beam of KEK-Accelerator Test Facility [20]. We have measured OTR in the PWZ and confirmed the characteristics presented in [18].

We have also developed and applied a method for PWZ suppression valid for optical wavelengths. The theory predicted that, by putting the detector in the back focal plane of a focusing lens, the PWZ effect should disappear. Moreover, according to [19] all DR models developed in [21-24] should be valid.

Furthermore, a method for prewave zone suppression applicable for longer radiation wavelengths is discussed.

\section{DIFFRACTION RADIATION IN THE PREWAVE ZONE}

Diffraction radiation (DR) appears when a charged particle moves in the vicinity of a medium (target). The field of passing particle interacts with the target's surface inducing currents changing in time. Those currents give rise in radiation. The DR photon yield becomes significant and easily detectable if the impact parameter, $h$ (the shortest distance between the target and the particle trajectory), is smaller than the effective electron field radius:

$$
h \leq \frac{\gamma \lambda}{2 \pi} .
$$

In this case the electron does not directly interact with the target medium. It excludes multiple scattering of the incident particle on the electron shells of the medium atoms. It precludes both the target damage due to the ionization heating process and the worsening of the electron beam parameters. That is what makes the DR phenomenon very interesting.

The PWZ theory has been considered in many papers [11-16,19]. In this chapter we introduce the theoretical approach used for calculating the DR characteristics and for their comparison with experimental results. General formulas and the most important DR characteristics in both the prewave and far-field zones are described.

\section{A. General formulas}

Classic DR theory is based on Huygen's principle of diffraction of light [17]. The particle field is introduced as a superposition of the pseudophoton fields. When the particle passes nearby a flat screen, the pseudophotons are scattered from it converting into real ones, and propagate in the direction of specular reflection.

The calculation geometry is represented in Fig. 1. An electron moving rectilinearly and with constant velocity transverses a screen plane through a rectangular hole
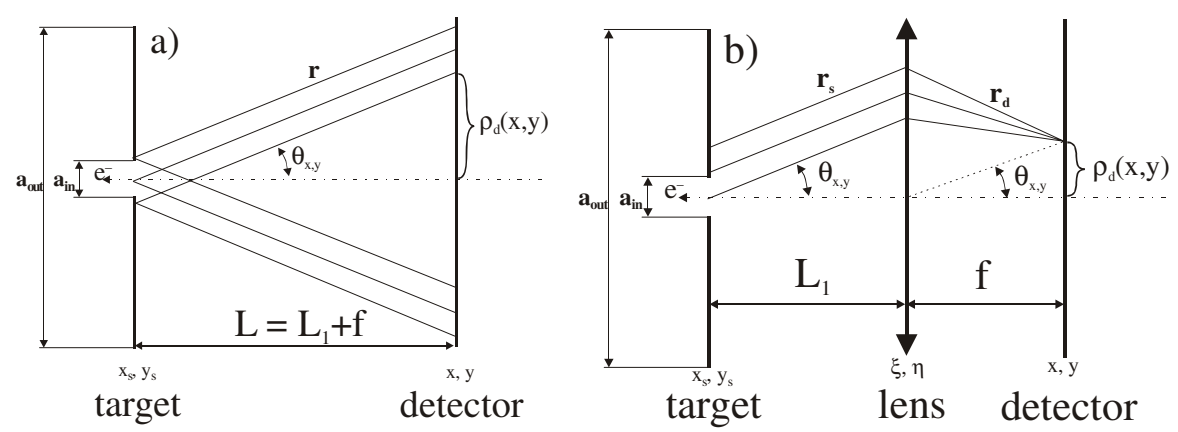

FIG. 1. Geometry of the DR production: (a) without the lens; (b) with the lens. Here $x_{s}$ and $y_{s}$ are the coordinates of the target surface; $\xi$ and $\eta$ are the coordinates of the lens surface; $x$ and $y$ are the detector coordinates; $L_{1}$ is the distance from the target to the lens; $f$ is the lens focus, $L=L_{1}+f$ is the distance from the target to the detector; $\theta_{x, y}$ are the radiation propagation angles measured from the specular reflection direction, $a_{\text {in }}$ and $a_{\text {out }}$ are the slit and screen dimensions along the $y$-axis, respectively. In the paper we also use the slit and screen dimensions in the perpendicular direction along the $x$-axis named as $b_{\text {in }}$ and $b_{\text {out }}$, respectively. 
placed perpendicular to the particle trajectory. In fact in our experiment the target is placed at a 45 deg angle with respect to the electron trajectory; however, a detailed analysis shows that, as long as the target tilt angle is much larger than $1 / \gamma$, the characteristics of DR propagating in the direction of specular reflection are not significantly different from a perpendicular case. In order to simplify the mathematical part of the paper, we shall use a perpendicular geometry and make all necessary links to the tilted target.

Throughout the paper the system of units $\hbar=m_{e}=c=$ 1 is used.

A general form of the DR field from an electron moving in the vicinity of a screen can be represented as

$$
E_{x, y}^{\mathrm{DR}}=-\frac{R_{x, y} k}{4 \pi^{2}} \iint E_{x, y}^{i}\left(x_{s}, y_{s}\right) \frac{e^{i \varphi}}{|r|} d y_{s} d x_{s} .
$$

Here the indices $x$ and $y$ represent the horizontal and vertical polarization components, $\varphi=k|r|$ is the phase advance of the waves propagating from the target to the detector, $r$ is the distance from an arbitrary radiation point on the target to an arbitrary point in the detector plane [see Fig. 1(a)], $k=2 \pi / \lambda$ is the wave number, $R_{x, y}$ are the Fresnel reflection coefficients, $E_{x, y}^{i}\left(x_{s}, y_{s}\right)$ is the amplitude of an arbitrary elementary source on the target surface, which is represented as an inverse Fourier transform of the incident particle field:

$$
\begin{aligned}
E_{x, y}^{i}\left(x_{s}, y_{s}\right) & =-\frac{i e}{2 \pi^{2}} \iint \frac{k_{x, y}^{\prime} \exp \left[i\left(k_{x}^{\prime} x_{s}+k_{y}^{\prime} y_{s}\right)\right]}{k_{x}^{\prime 2}+k_{y}^{\prime 2}+k^{2} \gamma^{-2}} d k_{x}^{\prime} d k_{y}^{\prime} \\
& =\frac{e k}{\pi \gamma} \frac{x_{s}, y_{s}}{\sqrt{x_{s}^{2}+y_{s}^{2}}} K_{1}\left(\frac{k}{\gamma} \sqrt{x_{s}^{2}+y_{s}^{2}}\right) .
\end{aligned}
$$

Here $e$ is the electron charge and $K_{1}$ is the McDonald function (or modified Bessel function). In Eq. (4) the double integral defines the dimensions and configuration of the screen.

The phase advance, $\varphi$, is determined by the time that photons need to reach the detector [see Fig. 1(a)]. Therefore, the phase term in Eq. (4) can be written as

$$
\frac{e^{i \varphi}}{|r|}=\frac{e^{i k|r|}}{|r|}=\frac{\exp \left[i k \sqrt{L^{2}+\left(x_{s}-x\right)^{2}+\left(y_{s}-y\right)^{2}}\right]}{\sqrt{L^{2}+\left(x_{s}-x\right)^{2}+\left(y_{s}-y\right)^{2}}} .
$$

This is the most general representation that includes a near-field effect. Assuming that the detector is placed at a distance much larger than the radiation source size determined by the effective electron field radius, we can use a series expansion and significantly simplify the phase term

$$
\frac{e^{i \varphi}}{|r|} \approx \frac{1}{L} \exp \left[\frac{i k}{2 L}\left(x_{s}^{2}+y_{s}^{2}\right)-\frac{i k}{L}\left(x_{s} x+y_{s} y\right)\right] .
$$

Here we omitted the phase terms independent of the integrals. One may see that the second term is responsible for the Fraunhofer diffraction. The first term is the first order Fresnel correction to the Fraunhofer diffraction theory. If the first term is negligibly small, the Eq. (7) represents a pure Fraunhofer diffraction case, i.e., the far-field approximation.

Let us consider the first term in Eq. (7). From the physical aspects of the DR process, we know that the amplitude of the elementary sources on the target surface at the distance much larger than the effective electron field radius is very small. That means that we can consider the range $x_{s}, y_{s}<\gamma \lambda / 2 \pi$. Therefore, the condition for the farfield approximation can be represented in the following form [19]:

$$
\frac{k}{2 L}\left(x_{s}^{2}+y_{s}^{2}\right) \ll 1 \Rightarrow \frac{\gamma^{2} \lambda}{2 \pi L} \ll 1 \Rightarrow L \gg \frac{\gamma^{2} \lambda}{2 \pi} .
$$

One may see that the condition (8) fully coincides with Eq. (2), that confirms its validity. If the condition represented by Eq. (8) is fulfilled, then one can consider the DR process in the far field. In this case the mathematical representation is significantly simplified ([21-24], and references therein).

The purpose of this work is to investigate the DR characteristics inside the prewave zone. In order to calculate the DR differential spatial-spectral distribution per unit photon energy and per unit angular interval including the PWZ effect, one should substitute Eq. (4) together with Eqs. (5) and (7) into

$$
\frac{d^{2} W^{\mathrm{DR}}}{d \omega d \Omega}=4 \pi^{2} L^{2}\left[\left|E_{x}^{\mathrm{DR}}\right|^{2}+\left|E_{y}^{\mathrm{DR}}\right|^{2}\right]
$$

Here $d \Omega=d x d y / L^{2}$ is the unit angular interval. One should keep in mind that $d \Omega=d \theta_{x} d \theta_{y}$ is valid in the farfield only. Moreover, in the far field Eq. (9) becomes a differential spectral-angular distribution, i.e., the angles of photon emission coincide with the observation angles.

The PWZ effect can be significantly suppressed or even rejected if the detector is placed in the back focal plane of a focusing lens [see Fig. 1(b)]. The expression for the DR field in the back focal plane of a lens can be written as (see, for instance, [19])

$$
\begin{aligned}
E_{x, y}^{\mathrm{DR}}= & \frac{i k^{2} R_{x, y}}{8 \pi^{3}} \iint d y_{s} d x_{s} \iint d \xi d \eta E_{x, y}^{i}\left(x_{s}, y_{s}\right) \\
& \times \frac{e^{i \varphi_{s}}}{\left|r_{s}\right|} e^{i \varphi_{l}} \frac{e^{i \varphi_{d}}}{\left|r_{d}\right|} .
\end{aligned}
$$

Here $\varphi_{s}=k\left|r_{s}\right|$ is the phase advance of the photons propagating from the target to the lens, $\varphi_{d}=k\left|r_{d}\right|$ is the phase advance of the photons propagating from the lens to the detector, and $\varphi_{l}$ is the phase defining the time delay caused by the lens. All these three phase terms can be represented in the following form: 


$$
\begin{aligned}
\frac{e^{i \varphi_{s}}}{\left|r_{s}\right|} & \approx \frac{1}{L_{1}} e^{\left[\left(i k / 2 L_{1}\right)\left(x_{s}^{2}+y_{s}^{2}\right)-\left(i k / L_{1}\right)\left(x_{s} \xi+y_{s} \eta\right)+\left(i k / 2 L_{1}\right)\left(\xi^{2}+\eta^{2}\right)\right]} \\
e^{i \varphi_{l}} & \approx e^{\left[-(i k / 2 f)\left(\xi^{2}+\eta^{2}\right)\right]} \\
\frac{e^{i \varphi_{d}}}{\left|r_{d}\right|} & \approx \frac{1}{f} e^{\left[(i k / 2 f)\left(x^{2}+y^{2}\right)-(i k / f)(x \xi+y \eta)+(i k / 2 f)\left(\xi^{2}+\eta^{2}\right)\right]}
\end{aligned}
$$

The phase term, $\varphi_{l}$, is presented in a thin lens approximation. One should know that in the approach presented in this paper the optical axis of the lens coincides with the electron beam trajectory. However, in reality an electron beam has finite transverse dimensions. In this case some part of the beam electrons has offsets with respect to the optical axis. Nevertheless, for a thin lens approximation and for the detector placed in lens focus, those offsets do not cause any difference and can be forgotten. This approximation is valid in our case because the distances in our experiment are much larger than the cross section of the DR light beam at any location of the optical system. Substituting Eq. (11) in (10) and omitting the phase terms independent of the integrals, the DR field at the detector [see Fig. 1(b)] can be written as

$$
\begin{aligned}
E_{x, y}^{\mathrm{DR}}= & \frac{i k^{2} R_{x, y}}{L_{1} f 8 \pi^{3}} \iint d y_{s} d x_{s} E_{x, y}^{i}\left(x_{s}, y_{s}\right) \\
& \times \exp \left[-i k\left(x_{s} \frac{x}{f}+y_{s} \frac{y}{f}\right)\right] \\
& \times \int \exp \left[\frac{i k}{2 L_{1}}\left(\xi-x_{s}-x \frac{L_{1}}{f}\right)^{2}\right] d \xi \\
& \times \int \exp \left[\frac{i k}{2 L_{1}}\left(\eta-y_{s}-y \frac{L_{1}}{f}\right)^{2}\right] d \eta .
\end{aligned}
$$

The last two integrals in Eq. (12) define the lens shape and take into account the diffraction of DR light on the lens of finite dimensions. According to [19], one can apply an infinite lens approximation, if the following condition is fulfilled:

$$
R_{l} \gg \frac{L_{1}}{\gamma}+\frac{\gamma \lambda}{2 \pi}
$$

Here $R_{l}$ is lens radius. In this case the expression (12) can be significantly simplified:

$$
\begin{aligned}
E_{x, y}^{\mathrm{DR}}= & -\frac{R_{x, y} k}{f 4 \pi^{2}} \iint d y_{s} d x_{s} E_{x, y}^{i}\left(x_{s}, y_{s}\right) \\
& \times \exp \left[-i k\left(x_{s} \frac{x}{f}+y_{s} \frac{y}{f}\right)\right] .
\end{aligned}
$$

One may see that Eq. (14) represents the DR field in the form similar to the far-field (Fraunhofer diffraction) case. By making the following substitution,

$$
\frac{x}{f} \rightarrow \theta_{x} \quad \text { and } \quad \frac{y}{f} \rightarrow \theta_{y} .
$$

One can derive models used in [21-25] to calculate DR characteristics in the far-field zone. Equation (14) demonstrates that a thin lens can remove the PWZ effect.

In order to calculate spatial-spectral characteristics of DR in a back focal plane of a lens, one should use an expression similar to Eq. (9):

$$
\frac{d^{2} W^{\mathrm{DR}}}{d \omega d \Omega}=4 \pi^{2} f^{2}\left[\left|E_{x}^{\mathrm{DR}}\right|^{2}+\left|E_{y}^{\mathrm{DR}}\right|^{2}\right] .
$$

Here $d \Omega=d x d y / f^{2}$ is the unit angular interval measured in the space behind the lens [see Fig. 1(b)].

One should say that in a real experimental situation chromatic aberrations in a lens might introduce an additional systematic error. In order to minimize that, one should choose the lens focus to be much larger than the transverse dimensions of the radiation spot on the lens surface. In this case the chromatic aberrations can be neglected.

\section{B. Far-field transition (TR) and diffraction (DR) radiation properties}

The far-field DR as a possible tool for noninvasive electron beam diagnostics has been considered in detail during the past few years [21-25]. An expression for radiation field in far-field zone can be represented in the following way:

$$
\begin{aligned}
E_{x, y}^{\mathrm{DR}}= & -\frac{i e k^{2} R_{x, y}}{L 4 \gamma \pi^{3}} \iint d y_{s} d x_{s} \frac{x_{s}, y_{s}}{\sqrt{x_{s}^{2}+y_{s}^{2}}} K_{1}\left(\frac{k}{\gamma} \sqrt{x_{s}^{2}+y_{s}^{2}}\right) \\
& \times \exp \left[-i k\left(x_{s} \theta_{x}+y_{s} \theta_{y}\right)\right] .
\end{aligned}
$$

This expression can be easily derived using Eqs. (4) and (7) by applying the far-field condition (8). Here $\theta_{x, y}=$ $\arctan (x, y / L) \approx x, y / L$.

The integral in the Eq. (17) defines the target profile. By choosing infinite integral limits, one can derive a wellknown expression for the backward TR field from a charged particle crossing an infinite boundary between vacuum and a medium:

$$
\left(\begin{array}{c}
E_{x}^{\mathrm{inf}} \\
E_{y}^{\mathrm{inf}}
\end{array}\right)=\frac{i e}{2 \pi^{2} L}\left(\begin{array}{l}
R_{x} \theta_{x} \\
R_{y} \theta_{y}
\end{array}\right) \frac{1}{\theta_{x}^{2}+\theta_{y}^{2}+\gamma^{-2}} .
$$
tion

Applying Eq. (9), we have TR spectral-angular distribu-

$$
\frac{d^{2} W^{\mathrm{inf}}}{d \omega d \Omega}=\frac{\alpha}{\pi^{2}} \frac{\left|R_{x}\right|^{2} \theta_{x}^{2}+\left|R_{y}\right|^{2} \theta_{y}^{2}}{\left(\theta_{x}^{2}+\theta_{y}^{2}+\gamma^{-2}\right)^{2}} .
$$

Here $\alpha$ is the fine structure constant. Figure 2(a) represents a three-dimensional TR angular distribution. Actually the most popular TR angular distribution is an azimuthally symmetric ("crater"-like) one. However, that is only valid for either a perfectly conducting target material or for a normal particle incidence. One may see that the TR has an azimuthal asymmetry in Fig. 2(a). The reason is 

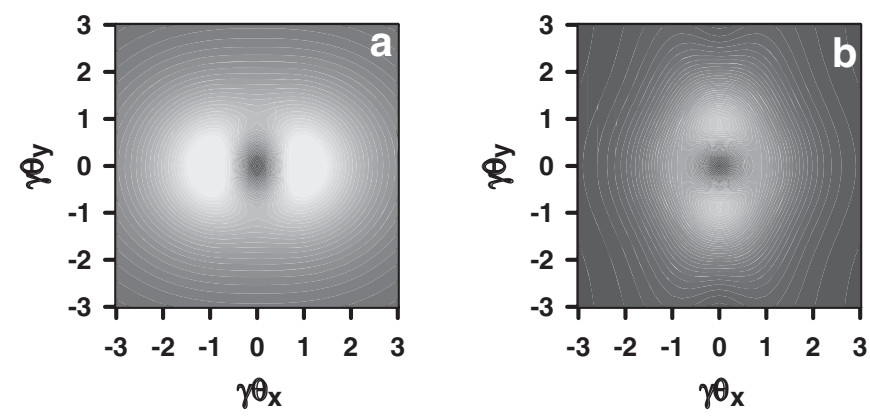

FIG. 2. (a) Angular distribution of TR from a particle crossing an infinite boundary. (b) Angular distribution of DR from a particle moving through a slit between two semiplanes. Calculation parameters: $\gamma=2500 ; \lambda=500 \mathrm{~nm} ; \quad a_{\text {in }}=$ $0.185 \mathrm{~mm}$ and $a_{s}=0$; reflection coefficients for gold, $\theta_{0}=$ $45^{\circ}$ target tilt angle and $500 \mathrm{~nm}$ wavelength are $\left|R_{x}\right|^{2}=0.61$ and $\left|R_{y}\right|^{2}=0.37[26,27]$.

that, if the target is tilted with respect to the electron trajectory, the Fresnel reflection coefficients (that take into account the medium properties [26,27]) for vertical and horizontal polarization components might be significantly different. The difference is determined by the target tilt angle, the radiation wavelength, and the target material. This phenomenon was clearly observed in [28]. As it is described in the next section, our target is a silicon wafer covered with gold; therefore, all calculations in the theoretical part of the paper are performed for a gold target.

Integrating Eq. (17) from $-\infty$ to $\infty$ over $x_{s}$, from $-\infty$ to $-a_{\text {in }} / 2+a_{s}$, and from $a_{\text {in }} / 2+a_{s}$ to $\infty$ over $y_{s}$ [where $a_{\text {in }}$ and $a_{s}$ are the slit size (see Fig. 1) and the offset of the electron trajectory with respect to the slit center, respectively], one may obtain an expression for two polarization components of DR spectral-angular distribution generated by a charged particle moving through a slit between two semiplanes [21-25]:

$$
\begin{aligned}
\frac{d^{2} W_{x}}{d \omega d \Omega}= & \frac{\alpha}{4 \pi^{2}}\left|R_{x}\right|^{2} \frac{\theta_{x}^{2}}{\gamma^{-2}+\theta_{x}^{2}} \frac{\exp \left(-\frac{2 \pi a_{\text {in }}}{\lambda} \sqrt{\gamma^{-2}+\theta_{x}^{2}}\right)}{\gamma^{-2}+\theta_{x}^{2}+\theta_{y}^{2}} \\
& \times\left\{\cosh \left[\frac{4 \pi a_{s}}{\lambda} \sqrt{\gamma^{-2}+\theta_{x}^{2}}\right]\right. \\
& \left.+\cos \left[\frac{2 \pi a_{\text {in }}}{\lambda} \theta_{y}+2 \psi\right]\right\} \\
\frac{d^{2} W_{y}}{d \omega d \Omega}= & \frac{\alpha}{4 \pi^{2}}\left|R_{y}\right|^{2} \frac{\exp \left(-\frac{2 \pi a_{\text {in }}}{\lambda} \sqrt{\gamma^{-2}+\theta_{x}^{2}}\right)}{\gamma^{-2}+\theta_{x}^{2}+\theta_{y}^{2}} \\
& \times\left\{\cosh \left[\frac{4 \pi a_{s}}{\lambda} \sqrt{\gamma^{-2}+\theta_{x}^{2}}\right]\right. \\
& \left.-\cos \left[\frac{2 \pi a_{\text {in }}}{\lambda} \theta_{y}+2 \psi\right]\right\} .
\end{aligned}
$$

Equation (20) represents DR spectral-angular distribution for a case when the particle trajectory is perpendicular to the target plane. According to [22], for a tilted case (if the target tilt angle is $\gg 1 / \gamma$ ) DR and TR distribution remain unchanged, except the angles $\theta_{x}$ and $\theta_{y}$ should be measured from the mirror reflection direction and the $a_{\mathrm{in}}$ is an effective slit size (the real slit size is determined as $a=$ $a_{\text {in }} / \sin \theta_{0}$, where $\theta_{0}$ is the target tilt angle measured from the electron trajectory).

DR three-dimensional angular distribution is illustrated in Fig. 2(b). This distribution has been measured experimentally and presented in $[28,29]$. The DR maximum intensity is comparable to the TR one. However, the most important far-field DR characteristics are represented in Fig. 3. One may see that if the electron moves off center of the slit the intensity increases because the particle moves closer to one of the target edges, but the distribution itself remains symmetric. That is because the distance from target to the observation point is large enough to accomplish the interference of the radiation produced at different parts of the target.

One may notice that Eq. (14) is similar to Eq. (17) used for the derivation of the far-field equations (18)-(20). That means that if the detector is placed in the back focal plane of a lens all DR and TR properties should be the same as in the far-field approach. In this case the angular variables should be $\theta_{x, y}=\operatorname{arctg}(x, y / f) \approx x, y / f$. The most important thing to know is that Eq. (14) is independent of distance from the target to the lens.

In the next section we describe both the basic TR and DR properties in the prewave zone and the problems related to it.

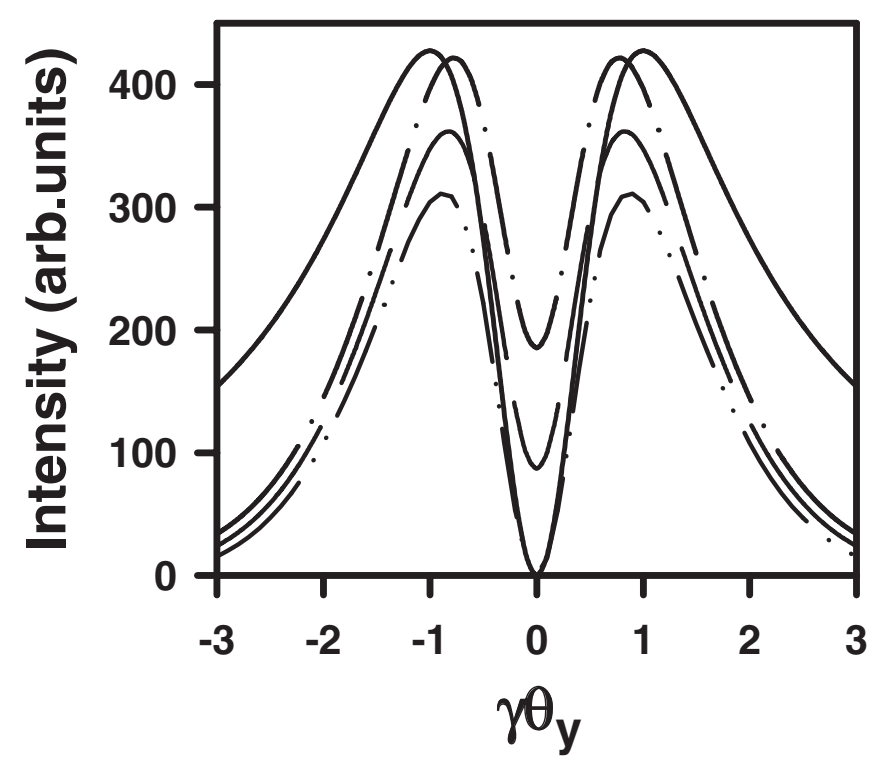

FIG. 3. TR (solid line) and DR angular distributions calculated for different electron positions with respect to the slit center: $a_{s}=0$, dash-dot-dotted line; $a_{s}=70 \mu \mathrm{m}$, dashed line; and $a_{s}=90 \mu \mathrm{m}$, dash-dotted line. Calculation parameters: $\gamma=$ 2500, $\lambda=500 \mathrm{~nm}, a_{\text {in }}=0.185 \mathrm{~mm}, \theta_{0}=45^{\circ}$, and $\left|R_{y}\right|^{2}=$ 0.37 . 


\section{Transition and diffraction radiation properties in the prewave zone}

The prewave zone (PWZ) effect in the TR phenomenon was predicted theoretically in [14]. Later it was observed experimentally in [18]. We assumed that the PWZ effect in the DR phenomenon could be similar or even more severe.

The TR field generated by a charged particle passing through a rectangular conducting screen in the PWZ can be obtained by substituting Eq. (7) in (4):

$$
\begin{aligned}
E_{x, y}^{\mathrm{TR}}\left(a_{\text {out }}, b_{\text {out }}\right)= & -\frac{R_{x, y} k}{L 4 \pi^{2}} \int_{-a_{\text {out }} / 2+a_{s}}^{a_{\text {out }} / 2+a_{s}} d y_{s} \\
& \times \int_{-b_{\text {out }} / 2+b_{s}}^{b_{\text {out }} / 2+b_{s}} d x_{s} E_{x, y}^{i}\left(x_{s}, y_{s}\right) \\
& \times e^{\left[(i k / 2 L)\left(x_{s}^{2}+y_{s}^{2}\right)-(i k / L)\left(x_{s} x+y_{s} y\right)\right]} .
\end{aligned}
$$

Here $a_{\text {out }}$ and $b_{\text {out }}$ are outer target dimensions (see Fig. 1); $a_{s}$ and $b_{s}$ are the offsets of the electron trajectory with respect to the target center along the $y$-axis and the $x$-axis, respectively. In order to avoid the effect of finite target dimensions, in Eq. (21) the following condition should be fulfilled:

$$
a_{\text {out }} \gg \frac{\gamma \lambda}{2 \pi} \quad \text { and } \quad b_{\text {out }} \gg \frac{\gamma \lambda}{2 \pi}
$$

This is the approximation of an infinite boundary.

Substituting Eq. (21) in (9), we can calculate TR spectral-spatial characteristics in the PWZ. The calculation results are represented in Fig. 4. Figure 4(a) represents the TR spatial distribution in the far-field zone $(L \gg$ $\left.\gamma^{2} \lambda / 2 \pi\right)$, which coincides with the distribution calculated with Eq. (19) and shown in Fig. 2(a). However, if the distance from target to detector is comparable to or smaller than the parameter $\gamma^{2} \lambda / 2 \pi$, one may see a serious broadening of the TR spatial distribution [see Figs. 4(c) and 4(d)]. Inside the PWZ the photon density is no longer inversely proportional to the squared distance from the source. Moreover, for higher electron beam energy the PWZ distance is longer. Figure 5(a) represents 2D TR distributions calculated for different distances and Fig. 5(b) shows the interpeak distance of the TR spatial distribution as a function of the target-to-detector distance. Since the distance, $L$, is represented in relative units, one can point out a region related to the optical wavelength range. In contrast to the far-field approach, the TR in the PWZ has an additional wavelength dependence that can be easily detected in an experiment. Furthermore, the interpeak distance in the far-field zone or in the back focal plane of a lens should be constant and equal to $\sim 2 \gamma^{-1}$.

This phenomenon in the incoherent part of the radiation spectrum was clearly observed in [18]. We have also begun our series of experiments with measurements of TR in the optical wavelength range, in order to confirm the results of [18] before starting DR measurements, which was expected to have a more complicated behavior.
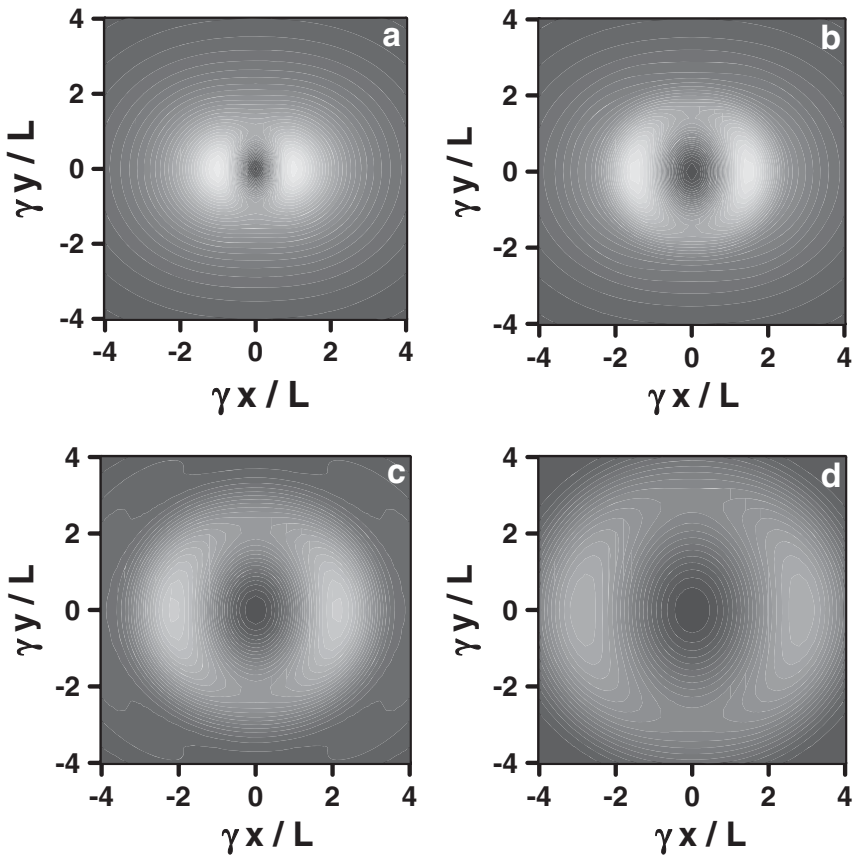

FIG. 4. TR 3D spatial distribution in the PWZ calculated for different distances from the target to the detector: (a) $L=$ $10 \gamma^{2} \lambda / 2 \pi=5 \mathrm{~m}$ (far-field zone), (b) $L=2 \gamma^{2} \lambda / 2 \pi=1 \mathrm{~m}$, (c) $L=0.9 \gamma^{2} \lambda / 2 \pi=0.45 \mathrm{~m}$, and (d) $L=0.5 \gamma^{2} \lambda / 2 \pi=$ $0.25 \mathrm{~m}$. Calculation parameters: $\gamma=2500, \lambda=500 \mathrm{~nm}, a_{\text {out }}=$ $9 \mathrm{~mm}, b_{\text {out }}=7 \mathrm{~mm}, a_{s}=b_{s}=0, \theta_{0}=45^{\circ},\left|R_{x}\right|^{2}=0.61$, and $\left|R_{y}\right|^{2}=0.37$.

The DR field generated by a charged particle passing through a rectangular opening in a rectangular screen in the PWZ can be calculated using well-known Babinet's principle. Applying Eq. (21), we have

$$
E_{x, y}^{\mathrm{DR}}\left(a_{\text {out }}, b_{\text {out }}\right)=E_{x, y}^{\mathrm{TR}}\left(a_{\text {out }}, b_{\text {out }}\right)-E_{x, y}^{\mathrm{TR}}\left(a_{\text {in }}, b_{\text {in }}\right) .
$$
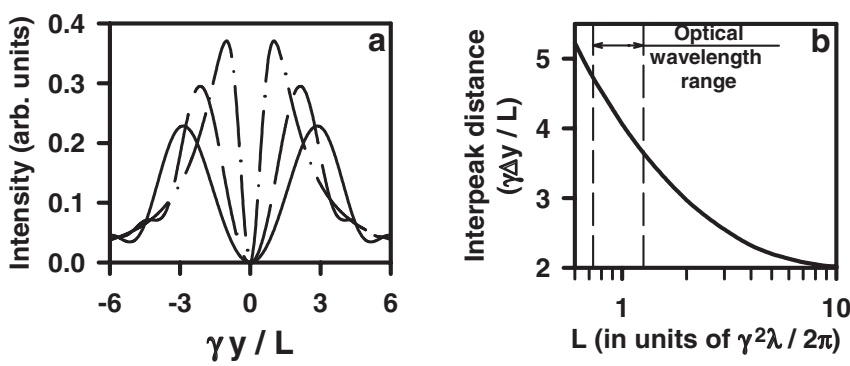

FIG. 5. (a) TR spatial distribution at $x=0$ in the PWZ calculated for different target-to-detector distances: $L=$ $10 \gamma^{2} \lambda / 2 \pi=5 \mathrm{~m} \quad$ (dash-dotted line), $L=0.9 \gamma^{2} \lambda / 2 \pi=$ $0.45 \mathrm{~m}$ (dashed line), and $L=0.5 \gamma^{2} \lambda / 2 \pi=0.25 \mathrm{~m}$ (solid line). (b) Interpeak distance calculated in units of $1 / \gamma$ as a function of the target-to-detector distance. Calculation parameters: $\gamma=2500, \lambda=500 \mathrm{~nm}, a_{\text {out }}=9 \mathrm{~mm}, b_{\text {out }}=7 \mathrm{~mm}, a_{s}=$ $b_{s}=0, \theta_{0}=45^{\circ}$, and $\left|R_{y}\right|^{2}=0.37$. Since the target-to-detector distance is represented in relative units, we can easily identify the range corresponding to the optical wavelength. 
One may calculate the DR spatial-spectral characteristics by substituting Eq. (23) in (9). Figure 6 represents the DR spatial distribution in the PWZ. One may see that as well as TR the DR distribution is also broader inside the PWZ. As well as TR, DR spatial distribution has a similar dependence as a function of the radiation wavelength or as a function of the target-to-detector distance. However, that is not the only peculiarity. If the electron passes through the center of the slit, one may see a symmetric distribution along the $x$-axis or the $y$-axis [Fig. 6(a)]. If there is an offset of the electron trajectory with respect to the slit center, a strong asymmetry appears, which is absent in the far-field zone (see, for instance, Fig. 3). Moreover, it becomes stronger while the offset increases. These DR radiation properties appear in the PWZ, where the distance from the source is not large enough, and interference from two halves of the target is not accomplished. If the offset, $b_{s}$, along the $x$-axis is also different from zero, the DR pattern has a lot more complicated structure.

Figure 7(a) illustrates the DR spatial distribution in the plane $x=0$ calculated for a few electron trajectory offsets with respect to the slit center. The asymmetry of the distribution can be calculated in the following manner:

$$
A=\frac{I_{\max }^{l}-I_{\max }^{r}}{I_{\max }^{l}+I_{\max }^{r}} 100 \% .
$$
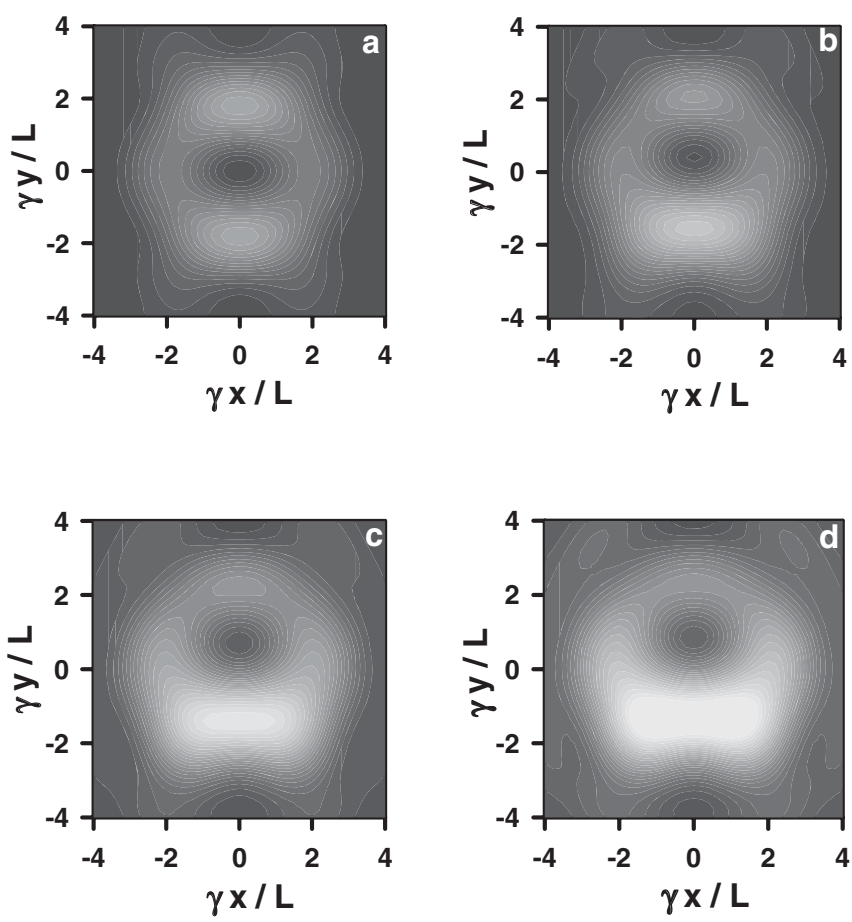

FIG. 6. DR 3D spatial distribution in the PWZ calculated for different offsets of the particle trajectory with respect to the slit center: (a) $a_{s}=0$, (b) $a_{s}=45 \mu \mathrm{m}, \quad$ (c) $a_{s}=75 \mu \mathrm{m}$, (d) $a_{s}=90 \mu \mathrm{m}$. Calculation parameters: $\gamma=2500, \lambda=$ $500 \mathrm{~nm}, a_{\text {out }}=9 \mathrm{~mm}, a_{\text {in }}=0.185 \mathrm{~mm}, b_{\text {out }}=7 \mathrm{~mm}, b_{\text {in }}=$ $5 \mathrm{~mm}, b_{s}=0, \theta_{0}=45^{\circ},\left|R_{x}\right|^{2}=0.61$, and $\left|R_{y}\right|^{2}=0.37$.
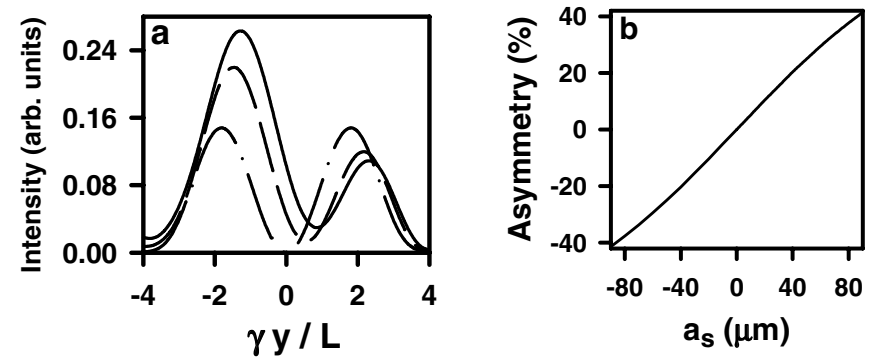

FIG. 7. (a) DR spatial distribution calculated for different electron beam trajectory offsets with respect to the target slit center: dash-dotted line $-a_{s}=0$, dashed line $-a_{s}=65 \mu \mathrm{m}$, and solid line $-a_{s}=90 \mu \mathrm{m}$. (b) DR spatial distribution asymmetry as a function of the electron offset. Calculation parameters: $\gamma=2500, \lambda=500 \mathrm{~nm}, a_{\text {out }}=9 \mathrm{~mm}, a_{\text {in }}=0.185 \mathrm{~mm}$, $b_{\text {out }}=7 \mathrm{~mm}, b_{\text {in }}=5 \mathrm{~mm}, b_{s}=0, \theta_{0}=45^{\circ},\left|R_{x}\right|^{2}=0.61$, and $\left|R_{y}\right|^{2}=0.37$.

Here $I_{\max }^{l}$ and $I_{\max }^{r}$ are the left and right maxima of the DR spatial distribution. The asymmetry as a function of the offset is represented in Fig. 7(b). One should notice that the asymmetry becomes stronger at shorter wavelengths, at higher electron energy, or at smaller target-to-detector distances. Figure 7(b) was calculated for an optical wavelength. The asymmetry reaches $40 \%$ that should be possible to measure in an experiment.

\section{EXPERIMENTAL SETUP}

The experiment was carried out at KEK-Accelerator Test Facility (ATF). The ATF consists of a photocathode RF gun, an $80 \mathrm{MeV}$ preinjector linac, a $1.28 \mathrm{GeV}$ S-band linac, a transport line, a damping ring, and an extraction line. KEK-ATF is a world's unique facility, which is one of the most appropriate machines for testing different accelerator techniques and technologies [20]. It has an extremely low emittance and high brightness beam, which is required by the future Linear Collider project. The parameters of extracted electron beam are introduced in Table I.

Our experimental setup was installed at the diagnostics section of the ATF extraction line. The experimental layout is represented in Fig. 8. The experimental setup was initially constructed as an ODR monitor for beam size measurements. It was well described in $[5,28,29]$. Here we

TABLE I. KEK-Accelerator Test Facility beam parameters.

\begin{tabular}{|c|c|c|}
\hline \multirow{3}{*}{$\begin{array}{l}\text { Maximum energy } \\
\text { Energy spread } \\
\text { Beam emittance }\end{array}$} & & $1.28 \mathrm{GeV}(\gamma=2500)$ \\
\hline & & $0.08 \%$ \\
\hline & $\begin{array}{l}\text { Vertical } \\
\text { Horizontal }\end{array}$ & $\begin{array}{c}(1.5 \pm 0.25) \times 10^{-11} \mathrm{~m} \mathrm{rad} \\
(1.4 \pm 0.25) \times 10^{-9} \mathrm{~m} \mathrm{rad}\end{array}$ \\
\hline \multicolumn{2}{|c|}{ Extracted bunch length } & $\sim 9 \mathrm{~mm}(30 \mathrm{ps})$ \\
\hline \multicolumn{2}{|c|}{ Single bunch population } & $(1 \div 2) \times 10^{10} \mathrm{e}^{-}$ \\
\hline Beam size at ODR & $\begin{array}{l}\text { Vertical } \\
\text { Horizontal }\end{array}$ & $\begin{array}{l}\sigma_{y}<10 \mu \mathrm{m} \\
\sigma_{x}<30 \mu \mathrm{m}\end{array}$ \\
\hline
\end{tabular}




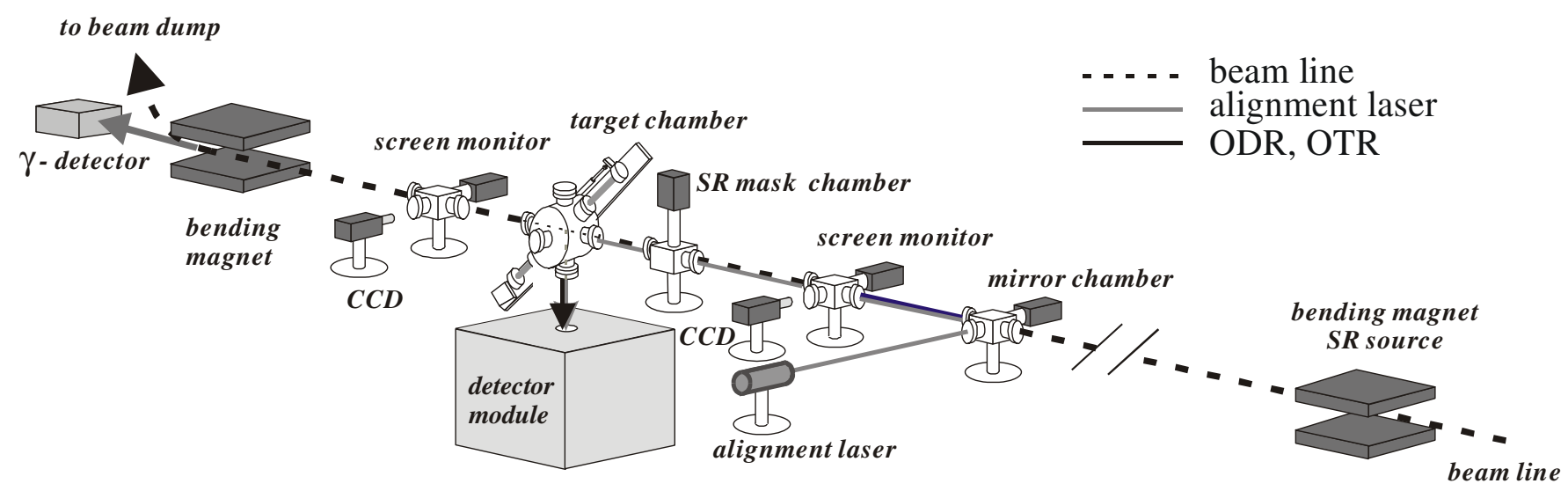

FIG. 8. Experimental layout.

briefly overview the main components of the ODR monitor and describe all modifications of the system we made for the prewave zone effect study.

The setup consists of a target vacuum chamber (ODR chamber), laser alignment system, and detector module. The target chamber design is based on the wire scanner used at KEK-ATF for beam size, emittance, dispersion, and jitter measurements [20]. It consists of a vacuum chamber, target holder mounted at $45 \mathrm{deg}$ with respect to the electron beam trajectory on a stage of remotely controlled pulse step motor. The linear gauge of the motor is $<0.5 \mu \mathrm{m}$.

The target was a silicon wafer plate coated with gold. The plate has a $5 \mathrm{~mm} \times 0.26 \mathrm{~mm}$ hole made by chemical etching. Since the horizontal hole width is much larger than the electron field radius treated as $\gamma \lambda / 2 \pi$, the target can be considered as a slit between two semiplanes. Furthermore, two halves of the target are not completely separated. Because of that two parts of the target are naturally coplanar. The target parameters are listed in Table II. The target configuration was described in [28]. Figure 9 represents the Fresnel reflection coefficients of gold calculated using $[26,27]$ for optical wavelength range and $45^{\circ}$ incidence angle. Namely, these values for reflection coefficients were used for ODR calculation and comparison with the experiment.

The detector module is represented in Fig. 10. As a detector we applied a highly sensitive cooled CCD camera system. The CCD parameters are listed in Table III. The

TABLE II. Target parameters.

\begin{tabular}{llc}
\hline \hline $\begin{array}{l}\text { Average roughness } \\
\text { Average flatness }\end{array}$ & $20 \mathrm{~nm}$ \\
Silicon dimensions & Thickness & $<100 \mathrm{~nm}$ \\
& Width $\left(b_{\text {out }}\right)$ & $0.3 \mathrm{~mm}$ \\
& Height $\left[a_{\text {out }} / \sin \left(45^{\circ}\right)\right]$ & $7 \mathrm{~mm}$ \\
Slit dimensions & Width $\left(b_{\text {in }}\right)$ & $9 \mathrm{~mm}$ \\
& Height $\left[a_{\text {in }} / \sin \left(45^{\circ}\right)\right]$ & $0.26 \mathrm{~mm}$ \\
Edge uniformity & & $\sim 1 \mu \mathrm{m}$ \\
Gold thickness & & $\sim 1 \mu \mathrm{m}$ \\
\hline \hline
\end{tabular}

CCD sensitivity as a function of wavelength is represented in Fig. 11.

In front of the CCD we installed a vertical stage to hold a polarizer, an optical filter, and a focusing lens. All those components can be easily removed or replaced when necessary. We used an optical lens with $f=200 \mathrm{~mm}$ and $400 \mathrm{~mm}$ in diameter. The lens-to-CCD distance was set to be $200 \pm 2 \mathrm{~mm}$, which is exactly the focal distance of the lens. Four optical filters were used in our experiment. The filter characteristics and Fresnel reflection coefficients for gold target are represented in Table IV.

The total distance from target to CCD sensor was $L=$ $440 \mathrm{~mm}$. For the wavelength range determined by our optical filters, the distance corresponds to $L=$ $(1.24 \sim 0.72) \gamma^{2} \lambda / 2 \pi$. According to the theoretical approach presented in the previous chapter, this distance range corresponds to a strong PWZ.

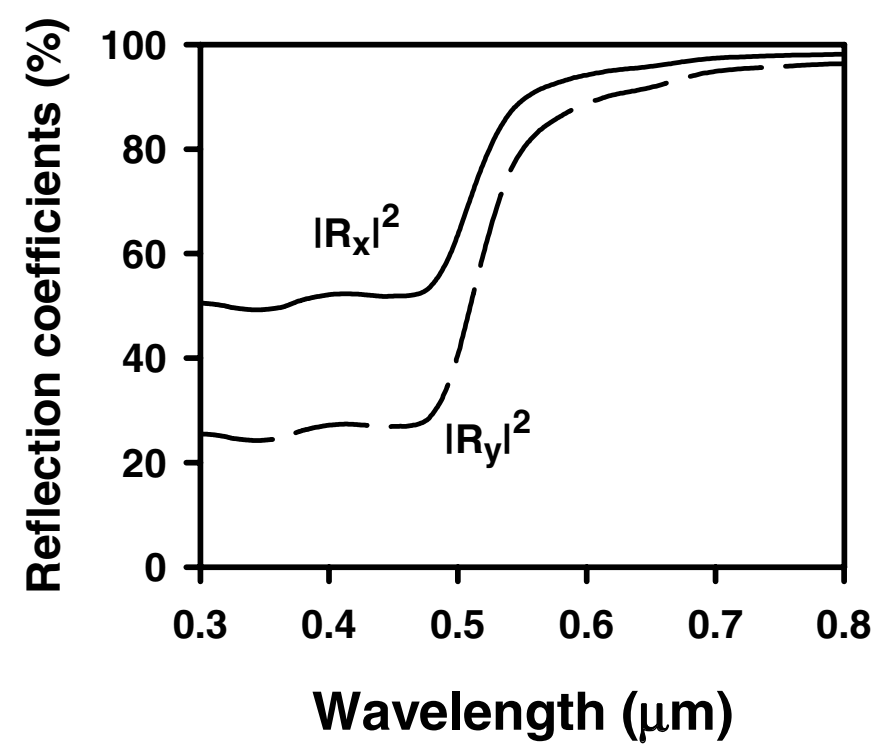

FIG. 9. Reflection coefficients for gold: vertical (dashed line) and horizontal (solid line) polarization components. 


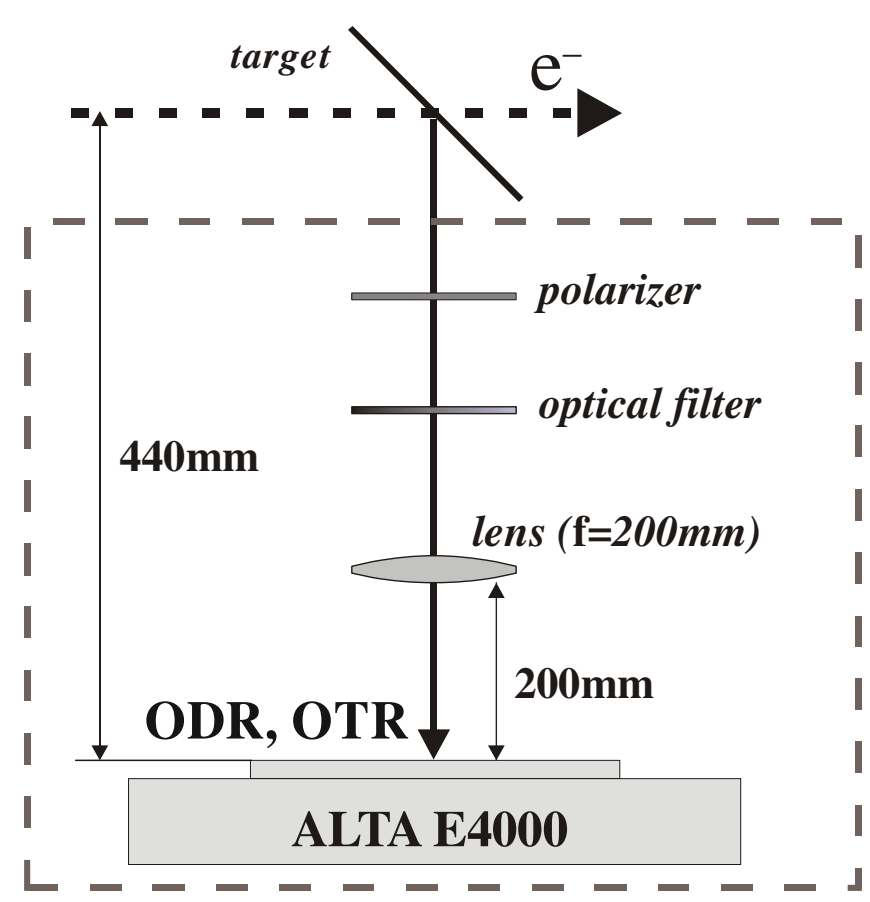

FIG. 10. Detector module.

The alignment system consists of a laser system, a vacuum mirror, and two screen monitors. The laser system consists of a laser stage, a He-Ne cw laser, a spatial filter, and a focusing lens. Changing the distance between the spatial filter and the lens, one may focus the laser at any point of the experimental setup (screen monitors, target, detector).

The vacuum mirror reflects the laser light along the beam line. We can observe the laser spot and electron beam spot at two screen monitors situated upstream and downstream of our target. If the laser position and electron beam position at those screens do not coincide, the laser direction can be corrected using precise adjustment screws of the laser stage. When the laser alignment system is tuned up, we low down the target; and reflecting the laser light from it, we can align all optical components of the detector module. The first experiment has shown that the alignment

TABLE III. Alta E4000-High performance cooled CCD camera system specifications.

\begin{tabular}{lc}
\hline \hline CCD type & Kodak KAI-4020M \\
Array size (pixels) & $2048 \times 2048$ \\
Pixel size & $7.4 \times 7.4$ microns \\
Imaging area & $15.15 \times 15.15 \mathrm{~mm}$ \\
Dynamic range & $>77 \mathrm{~dB}$ \\
Peak QE $(500 \mathrm{~nm})$ & $55 \%$ \\
Dark current & $0.2 \mathrm{e}^{-} / \mathrm{pixel} / \mathrm{sec} @-30^{\circ} \mathrm{C}$ \\
Digitization & $16 \mathrm{bit}$ \\
Minimum cooling temperature & $-30^{\circ} \mathrm{C}$ \\
Pixel binning & $1 \times 1$ to $10 \times 2048$ on-chip \\
\hline
\end{tabular}

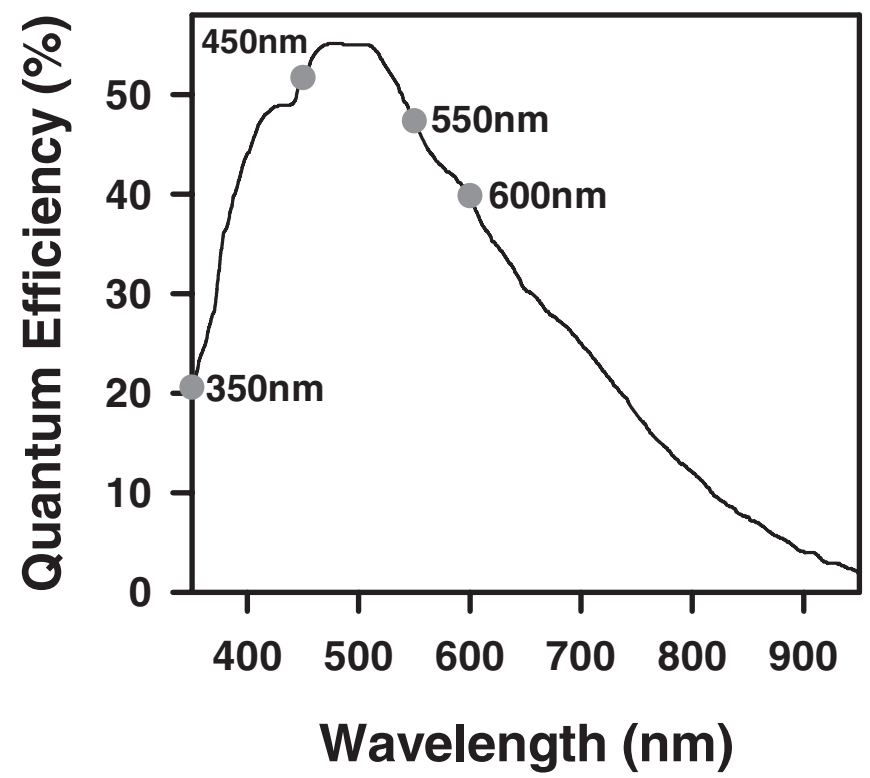

FIG. 11. Absolute quantum efficiency (QE) of the CCD sensor as a function of the detecting radiation wavelength. Gray points indicate the QE of the CCD sensor for central wavelengths of the optical filters used in the experiment.

system accuracy is smaller than $\gamma^{-1}=0.4 \mathrm{mrad}$, which is quite enough for our purposes.

A bending magnet situated $8 \mathrm{~m}$ upstream of our target was the main source of the synchrotron radiation (SR) background. In order to avoid SR background we set up a SR mask about $0.3 \mathrm{~m}$ upstream of our target. The mask performance has been described in details in [29]. The mask itself is a $20 \mathrm{~mm} \times 20 \mathrm{~mm}$ ceramic plate with a $1 \mathrm{~mm} \times 2 \mathrm{~mm}$ hole in it. This hole was big enough to let the electron beam go through without producing any significant ODR photons. Since the SR source is $8 \mathrm{~m}$ away, the SR spot in the vicinity of the target surface is rather large, and, therefore, the mask cuts off the dominant part of the SR background photons.

The mask chamber was mounted on a two-dimensional moving stage in order to adjust the mask position with respect to the electron beam. When the electron beam moves through the mask material it produces hard bremsstrahlung photons. Those photons were detected by an AirCherenkov counter installed downstream our target. Since the Air-Cherenkov detector registers very high-energy photons $(>20 \mathrm{MeV})$, the response from it strongly de-

TABLE IV. Parameters of optical filter.

\begin{tabular}{lrrrr}
\hline \hline \multicolumn{1}{c}{ Filter name } & $350 \mathrm{~nm}$ & $450 \mathrm{~nm}$ & $550 \mathrm{~nm}$ & $600 \mathrm{~nm}$ \\
\hline Center wavelength, nm & \multicolumn{1}{c}{354.7} & \multicolumn{1}{c}{458.7} & \multicolumn{1}{c}{558.2} & 608.8 \\
Peak transmission, \% & 30.15 & 65.96 & 68.26 & 60.70 \\
Bandwidth (FWHM), nm & 34.57 & 36.27 & 40.95 & 36.63 \\
$\left|R_{x}\right|^{2}$ for gold & 0.49 & 0.52 & 0.90 & 0.95 \\
$\left|R_{y}\right|^{2}$ for gold & 0.24 & 0.27 & 0.82 & 0.89 \\
\hline \hline
\end{tabular}




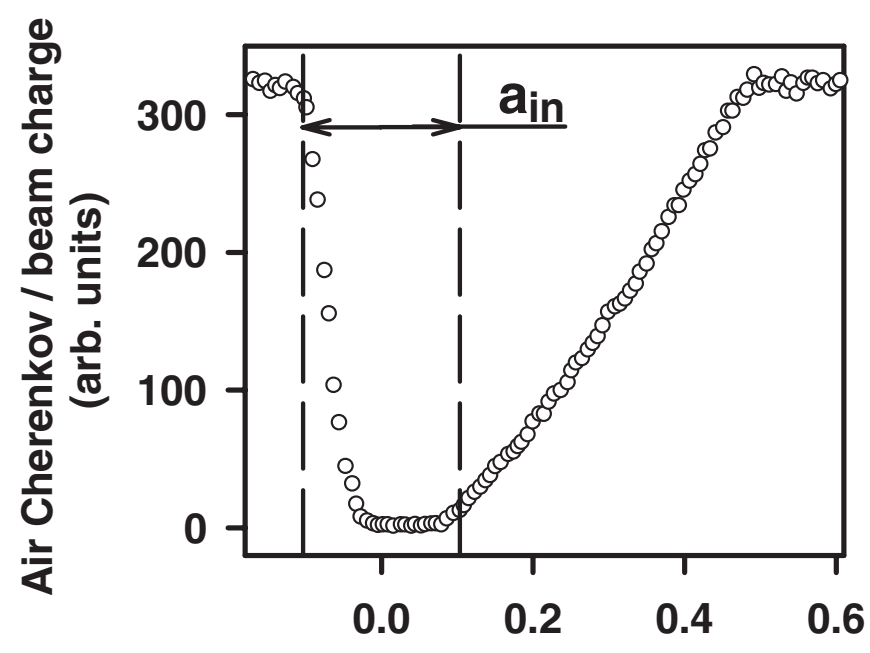

Target position $(\mathrm{mm})$

FIG. 12. Forward bremsstrahlung photon yield as a function of the target position normalized by the electron beam charge (open circles). Zero target position corresponds to the slit center and two dashed lines represent the effective slit width $\left(a_{\text {in }}\right)$.

pends on the number of particles directly interacting with the mask material. By measuring the photon yield as a function of the mask position, we could determine the mask hole center and let the electron beam go through it.

In a similar way we determined the ODR target position. A typical dependence of the bremsstrahlung photons as a function of the target position is represented in Fig. 12.

One may see that the dependence shown in Fig. 12 is asymmetric. The reason is that the target has oblique edges. A detailed description of the target design was represented in [28]. By making a linear fit of two straight sections, one may determine the slit size and its center with respect to the beam line.

We also have a $10 \mu \mathrm{m}$ tungsten wire mounted on the target holder for vertical beam size measurements. The measurement results are represented in the next chapter.

\section{EXPERIMENTAL RESULTS}

In order to achieve a success in our experiment, we optimized the electron beam optics using SAD code [30]. The purpose was to minimize the distortion of the OTR spatial distribution due to large transverse electron beam size that results in smoothing of the OTR pattern. The OTR radiation has an intrinsic angular divergence of order of $\gamma^{-1}$. In order to minimize the distortion, the beam size should obey the following inequality:

$$
\sigma_{x, y} \ll \frac{L}{\gamma}=176 \mu \mathrm{m}
$$

A similar distortion might be expected in the ODR spatial distribution together with the beam size effect considered in [21-25] and measured in [5].
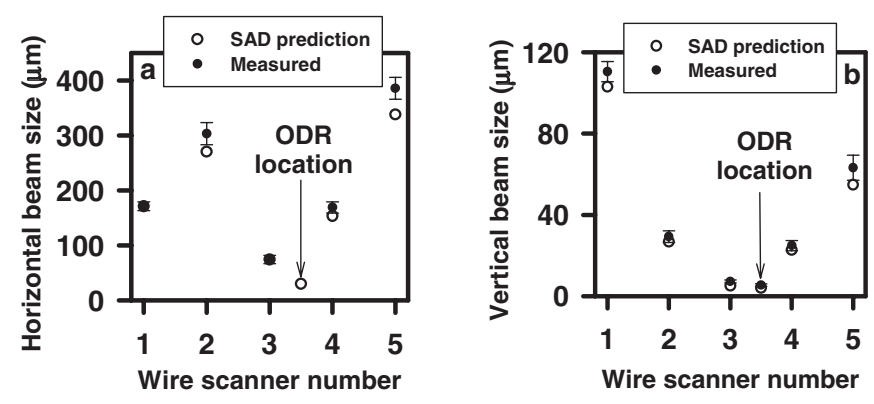

FIG. 13. Horizontal (a) and vertical (b) beam sizes measured with five wire scanners in the extraction line (black circles) and predicted with SAD code (open circles). The point between third and fourth wire scanners corresponds to the ODR target location. The vertical beam size was measured with $10 \mu \mathrm{m}$ tungsten wire.

The SAD calculations are demonstrated in Fig. 13 (open circles), where the horizontal scale is the wire scanner number at the ATF extraction line. The ODR chamber is located between the third and the fourth ones. The predicted beam sizes at the ODR target location were $\sigma_{x}=$ $30 \mu \mathrm{m}$ and $\sigma_{y}=4 \mu \mathrm{m}$.

The vertical beam size profile measured with the $10 \mu \mathrm{m}$ tungsten wire installed in the ODR chamber is represented in Fig. 14. The beam size determined from the Gaussian fit is bigger than the predicted one but it is small enough for our purposes. The difference between the calculation and measurements depends on how well the ATF damping ring is tuned up. It also depends on the electron beam charge since the intrabeam scattering effect was not taken into account in the calculations.

Unfortunately, we do not have a horizontal wire in the ODR chamber. However, we measured the horizontal beam size with five wire scanners in the extraction line situated upstream and downstream of our target. The calculations and measurements are consistent with each other. It al-

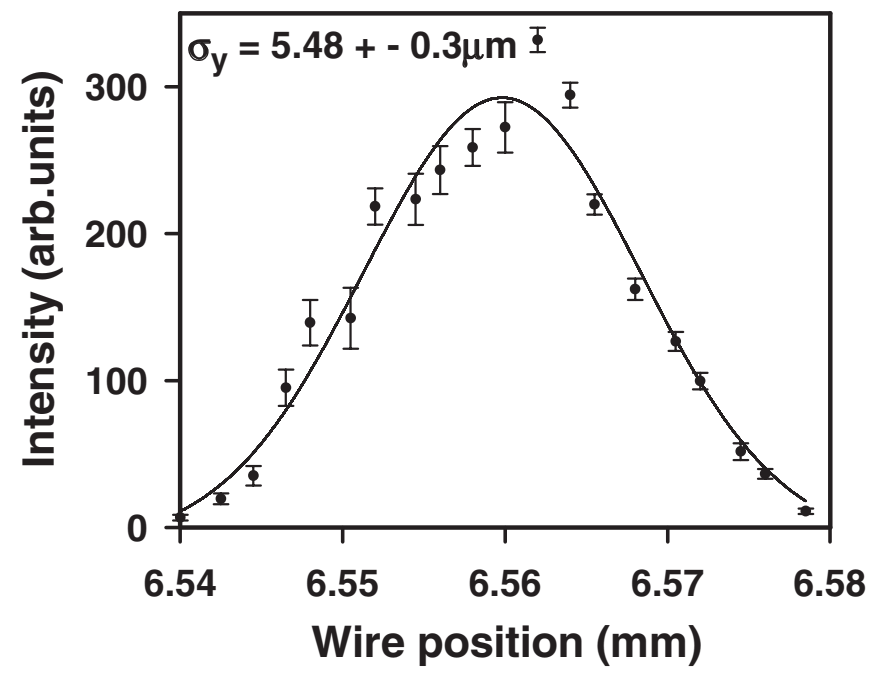

FIG. 14. Typical vertical beam profile measured with $10 \mu \mathrm{m}$ tungsten wire installed in the ODR chamber. 
lowed us to conclude that the horizontal beam size is also small enough.

One should say that, minimizing the transverse beam size, the beam angular divergence increases proportionally. It also might cause additional distortion of the OTR (ODR) spatial distribution. However, the ATF beam emittance is so small, that for our beam sizes the electron beam divergence is still much smaller than $\gamma^{-1}$, i.e., the smoothing of the radiation pattern is negligible.

\section{A. OTR measurements in the PWZ}

We started our experiment from observation of OTR spatial distribution. OTR phenomenon is well experimentally and theoretically studied, because of that we used OTR characteristics to verify our optical system and check any backgrounds interfering with later ODR measurements. Figure 15 demonstrates an OTR spatial distribution directly observed with the CCD camera. It is obvious that the OTR spatial distribution measured in the back focal plane of a lens [Fig. 15(b)] is by factor of 2 narrower, than the one measured without the lens [Fig. 15(a)]. The azimuthal asymmetry existing in both figures is determined by the difference in Fresnel reflection coefficients, which is consistent with the theoretical expectations [see, for instance, Figs. 2(a) and 4(c) corresponding to Figs. 15(a) and 15(b)].

However, according to the theoretical approach presented in Sec. II in the far-field zone, the spatial distributions measured with and without the lens should be exactly the same with the peak intensity at the polar angle of order of $1 / \gamma$. The PWZ effect results in broadening of the OTR spatial distribution, which is clearly seen in Fig. 15(a).

It is well known that the OTR spatial distribution is radially polarized. Therefore, as it was discussed in Sec. II, we can always represent the OTR spatial distribution as a sum of vertical and horizontal polarization components. The vertical polarization component was measured in the experiment by means of installing a polarizer in the optical path. The measured spatial distribution of the OTR vertical polarization component is represented in Fig. 16.
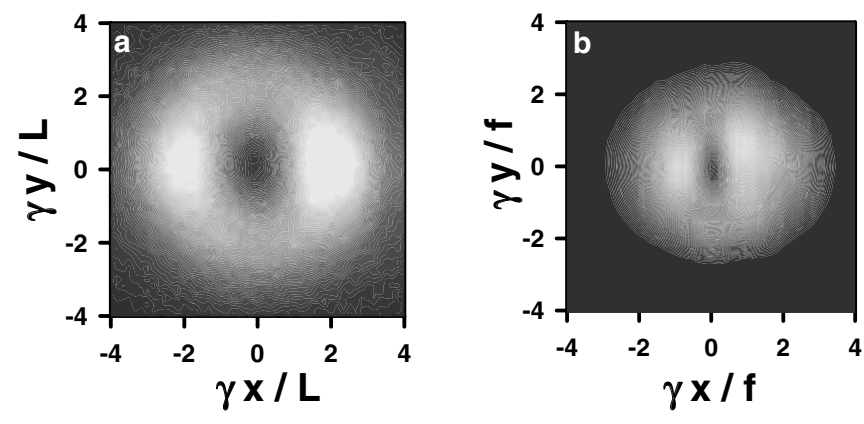

FIG. 15. OTR spatial distribution measured without (a) and with (b) the lens $(f=0.2 \mathrm{~m})$. Optical filter and polarizer were removed during these measurements.
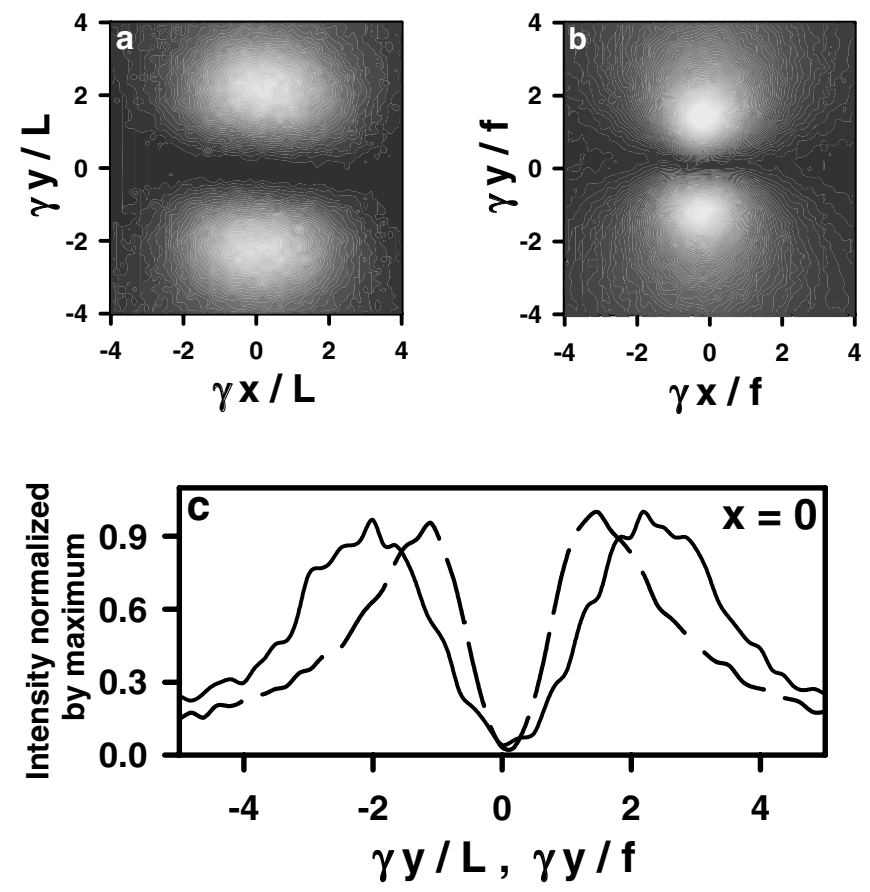

FIG. 16. Vertical polarization component of the OTR spatial distribution measured without (a) and with (b) the lens ( $f=$ $0.2 \mathrm{~m}$ ). (c) 2D OTR distributions in the plane $x=0$ measured without (solid line) and with (dashed line) the lens. An optical filter was removed during these measurements.

The theory also predicted a wavelength dependence of the OTR spatial distribution width (see, for instance, Fig. 5). Therefore, we have performed investigation of OTR spectral characteristics. Figure 17 illustrates the OTR spatial distribution measured with $450 \mathrm{~nm}$ optical filter.

One may see that the OTR distribution measured without the lens is about 2 times broader than the distribution measured with the lens. A similar broadening was observed in the previous experiment [18]. However, there was a weak doubt that the observed phenomenon was the prewave zone effect, because a certain target deformation might result in a similar broadening of the OTR spatial distribution. However, in the far-field zone a focusing lens should not change the OTR spatial distribution in the chosen angular units if the detector is in the back focal plane of the lens even in case of a deformed target. Moreover, the experiment and theory represented in Figs. 17(e) and 17(f) agree very well. Therefore, we conclude that the broadening of the OTR pattern is caused by the PWZ effect.

Figure 18(a) depicts the two-dimensional OTR spatial distribution measured with two different optical filters. Obviously the OTR distributions are different and in good agreement with the theoretically predicted ones represented in Fig. 18(b). The interpeak distance of the OTR spatial distribution measured with four optical filters is represented in Fig. 19. The wavelength dependence pre- 

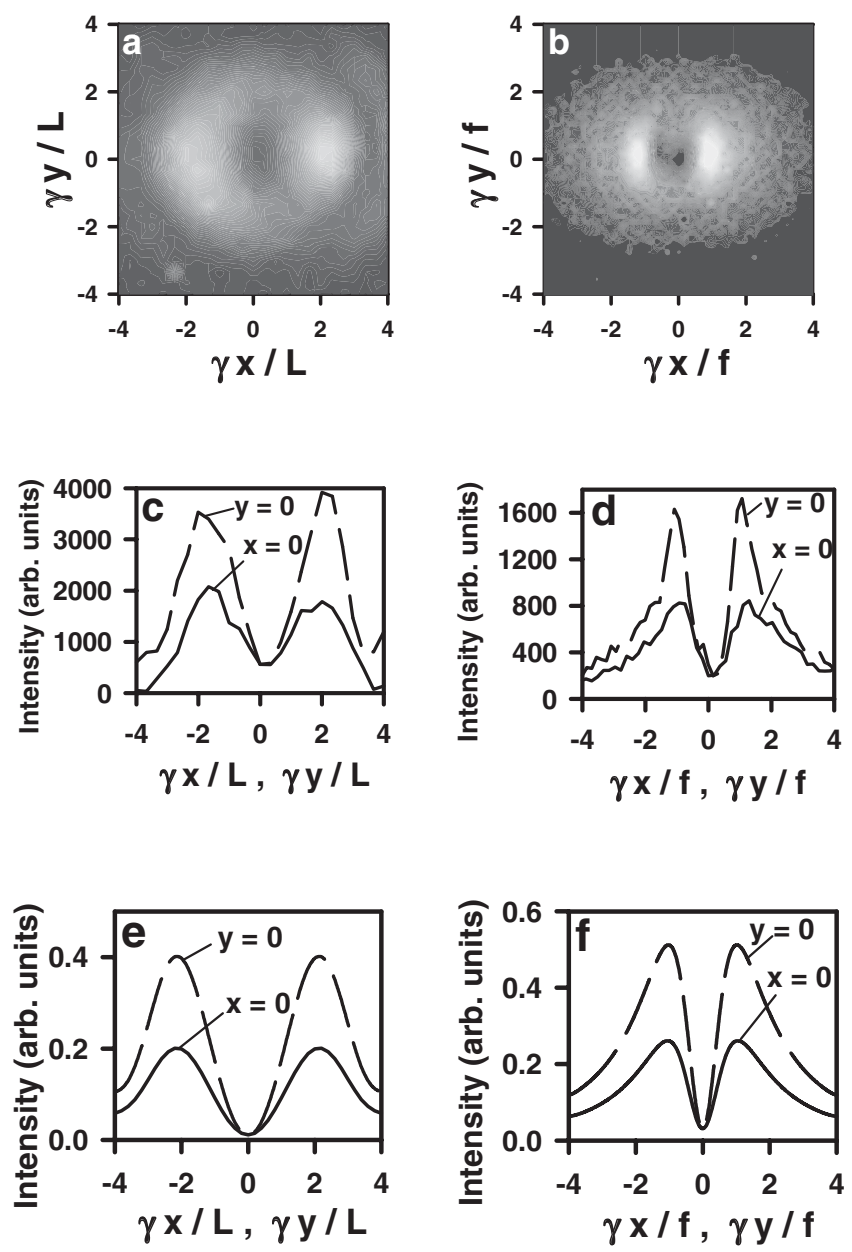

FIG. 17. OTR 3D spatial distributions measured with $450 \mathrm{~nm}$ filter without (a) and with (b) the lens $(f=0.2 \mathrm{~m})$; OTR 2D spatial distributions measured with $450 \mathrm{~nm}$ filter without (c) and with (d) the lens; OTR 2D spatial distributions calculated for $450 \mathrm{~nm}$ filter without (e) and with (f) the lens. The calculations were performed using Eq. (21) and the parameters were chosen to be the same as the experimental ones.

dicted by the theory is obvious. Moreover, the black circles in Fig. 19 demonstrate that, if the detector is placed in the back focal plane of the lens, the wavelength dependence disappears, i.e., the optical lens suppresses the PWZ effect.
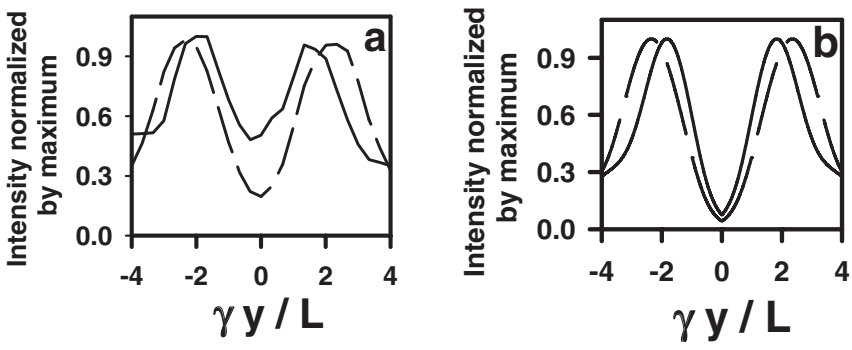

FIG. 18. (a) OTR 2D spatial distributions in the PWZ measured with $350 \mathrm{~nm}$ (solid line) and $600 \mathrm{~nm}$ (dashed line) filters without the lens $(f=0.2 \mathrm{~m})$. (b) OTR 2D spatial distributions calculated for $350 \mathrm{~nm}$ (solid line) and $600 \mathrm{~nm}$ (dashed line).

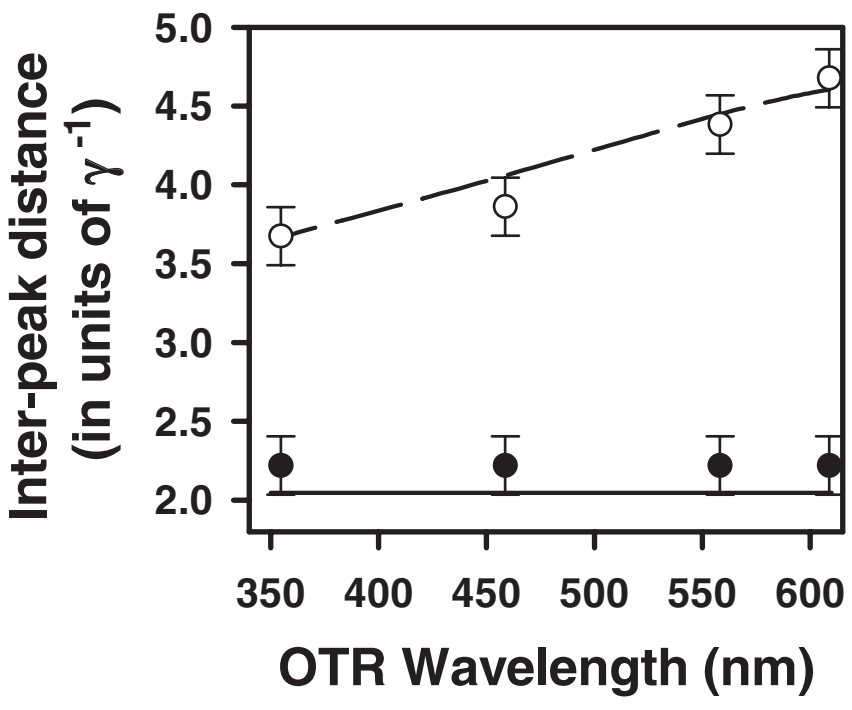

FIG. 19. Interpeak distance as a function of the radiation wavelength measured with (black circles) and without (open circles) the lens $(f=0.2 \mathrm{~m})$. Lines represent the theoretical prediction.

\section{B. Observation of the PWZ effect in the ODR phenomenon}

The key goal of the experiment was to observe and investigate the PWZ effect in ODR phenomenon. One should mention that all measurements in this chapter were performed with $550 \mathrm{~nm}$ optical filter. Moreover, in order to avoid significant SR contribution in the horizontal polarization component, we measured the ODR vertical polarization component only. Namely, the vertical polarization component of ODR carries the most valuable information.

Figure 20 demonstrates typical images of the ODR vertical polarization component measured without (a) and with (b) the lens with $f=200 \mathrm{~mm}$. As well as in the case of OTR presented in the previous section, ODR distributions are by a factor of 2 different in width. It is clear that the characteristics presented for OTR are present in the ODR phenomenon as well. However, the ODR has
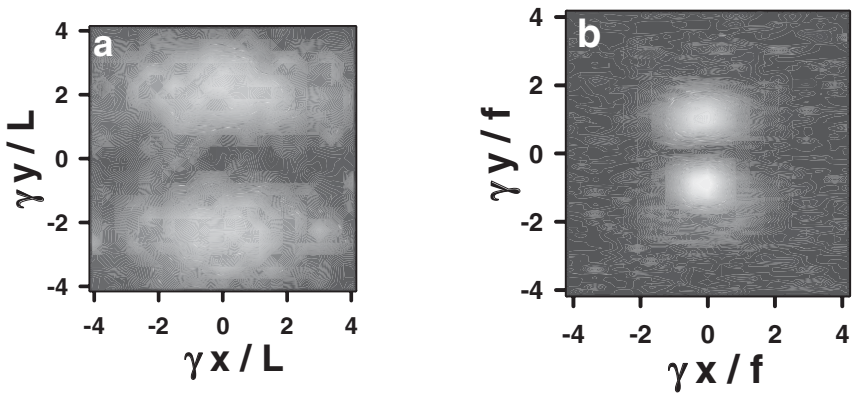

FIG. 20. Three-dimensional spatial distribution of ODR vertical polarization component measured without (a) and with (b) the lens and with $550 \mathrm{~nm}$ optical filter. 

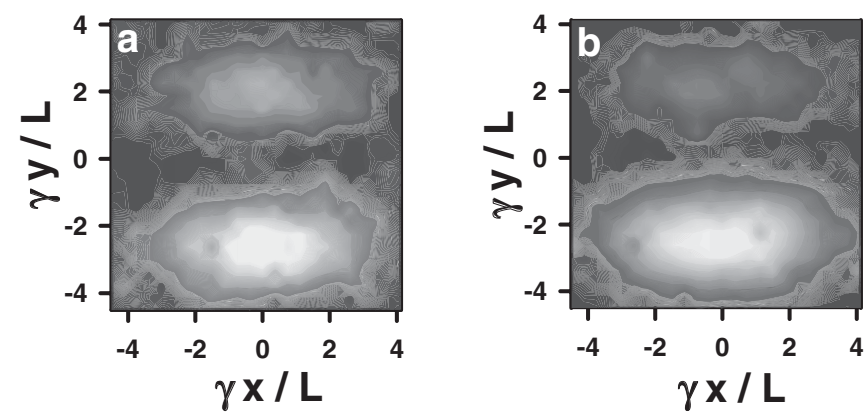

FIG. 21. Three-dimensional spatial distribution of ODR vertical polarization component measured without the lens and for two electron trajectory offsets with respect to the slit center: (a) $a_{s}=45 \mu \mathrm{m}$ and (b) $a_{s}=80 \mu \mathrm{m}$.

an additional peculiarity, i.e., it significantly depends on the electron beam trajectory offset with respect to the slit center.

Figure 21 shows two ODR spatial distributions measured for two different offsets. One may see that, if the electron beam does not move through the center of the target's slit, an asymmetry appears. Figure 22 demonstrates projections of ODR vertical polarization components onto the $y=0$ plane. The measurement results are in good agreement with calculations presented in the theoretical Sec. II. It is clear that the asymmetry increases when increasing the offset. The ODR in the far-field zone does not have this kind of peculiarity. Afterwards we installed

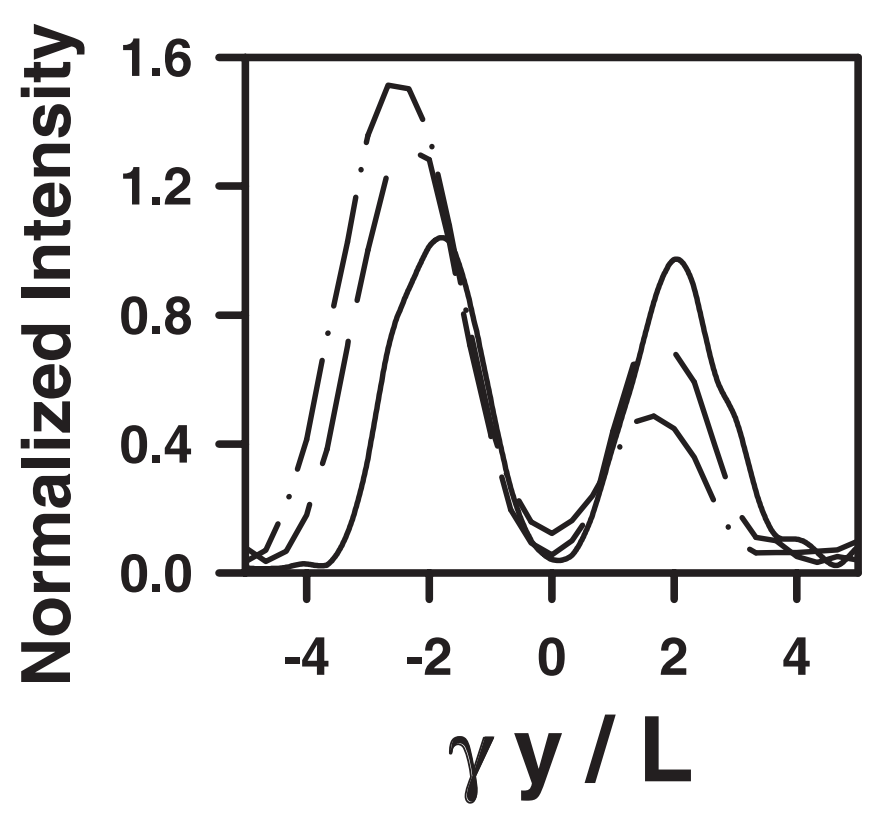

FIG. 22. Projection of ODR vertical polarization component measured without the lens for three different electron trajectory offsets with respect to the slit center: $a_{s}=0$ - solid line [Fig. 20(a)]; $a_{s}=45 \mu \mathrm{m}$-dashed line [Fig. 21(a)]; $a_{s}=80 \mu \mathrm{m}$-dash-dotted line [Fig. 21(b)]. the focusing lens into the optical system in order to check it.

According to the theory, if the detector is placed in the back focal plane of the lens the ODR characteristics should be the same as in the far-field zone. The results of the measurements with lens are represented in Fig. 23. One may see that the asymmetry almost disappeared. On the other hand when the lens is on, the intensity in the ODR distribution minimum increases with respect to the maximal intensity when shifting the electron beam trajectory with respect to the slit center, as it should be in the far-field zone (see Fig. 3). It happens because some part of the electron beam moves closer to one of the target edges. One should say that the beam moves at larger distance from the other edge. However, the contribution from the closer particles surpasses the contribution from the distant ones.

These characteristics have been already observed before by us in the far-field zone [5]. In this paper we represent the first observation of the ODR spatial distribution in the extreme PWZ but with far-field characteristics.

Figure 24 demonstrates a comparison of the asymmetry as a function of the electron beam trajectory offset with respect to the slit center measured with and without the lens. The lines represent the theoretically predicted dependences which agree well with the experiment especially at positive offsets. One may see a discrepancy between the experiment and the theory at negative offsets. We believe that the distortion of the ODR pattern is caused by a small contribution from the synchrotron radiation propagating
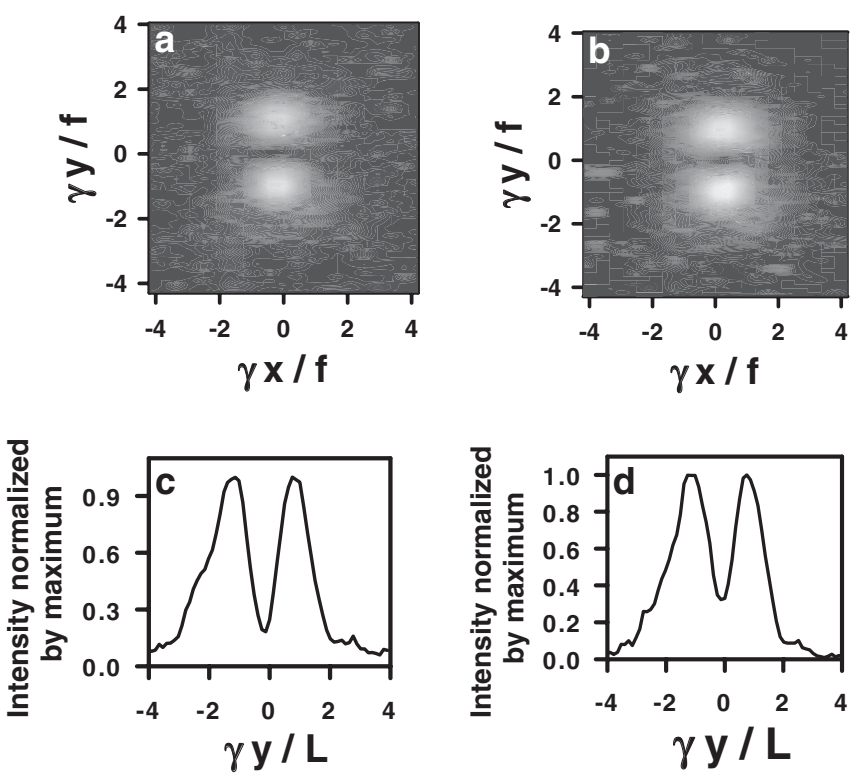

FIG. 23. Three-dimensional spatial distribution of ODR vertical polarization component measured with lens and for two electron trajectory offsets with respect to the slit center: (a) and (c) are for $a_{s}=45 \mu \mathrm{m}$ and its projection onto the $y=0$ plane, respectively; (b) and (d) are for $a_{s}=80 \mu \mathrm{m}$ and its projection onto the $y=0$ plane, respectively. 


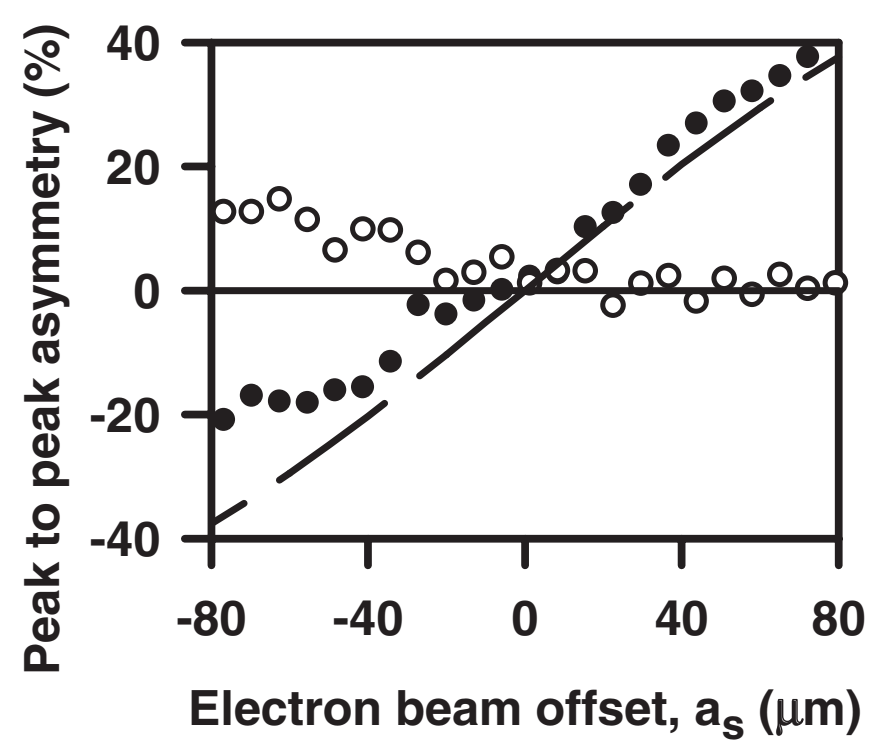

FIG. 24. Asymmetry of the ODR spatial distribution measured with (open circles) and without (black circles) the lens $(f=$ $0.2 \mathrm{~m}$ ). The lines represent theoretically predicted asymmetry calculated for experimental conditions and for two geometries: solid line — with the lens; and dashed line-without the lens.

through the mask hole and reflected from the target. By changing the position of target, its reflecting surface is changed, and, therefore, the amount of reflected SR is also changed. This discrepancy might be changed by adjusting the mask position with respect to the beam. However, it cannot be completely eliminated. Nevertheless, it is possible to minimize the SR contribution for the case when the electron beam is centered in the slit.

\section{CONCLUSION}

In this paper we represented observation and detailed investigation of the PWZ effect in optical transition (OTR) and diffraction (ODR) radiation phenomena.

We have confirmed results of the PWZ observation in OTR phenomenon performed in [18], i.e., we observed the broadening of the OTR spatial distribution and the width dependence on wavelength. The experiment is in good agreement with the theory.

We have demonstrated the first observation of the PWZ effect in the ODR phenomenon. ODR has specific peculiarity, i.e., dependence of its spatial distribution on the electron beam trajectory offset with respect to the slit center. We clearly observed the asymmetry predicted by the theory.

We have also demonstrated that if the detector is placed in the back focal plane of a focusing lens the PWZ effect can be eliminated. That is a good advantage of the method. The beam energy at KEK-ATF is definitely not the highest one achieved in the world. Since we could easily observe the PWZ effect, at higher energies it might cause serious problems. In our previous experiment [5,23], we increased the distance from target to detector in order to obey the farfield approximation. It was necessary in order to perform the beam size measurements. At the same time we complicated the optical system alignment that involved additional error in the experiment. For higher energy accelerators, the method of increasing the distance might not be suitable. For example, at SLAC FFTB (30 GeV beam), the far-field approximation requires the target-todetector distance to be as long as 1800 meters, which is practically impossible to achieve.

By putting the detector in the back focal plane of a lens it is possible to eliminate the PWZ effect and use all far-field methods [21-25] for beam diagnostics.

Beam size measurement was not the purpose of this paper. It will be considered in a later more proper one. However, the experimental setup described here is a good basis for a noninvasive single shot transverse beam size measurement because the optical system is much simpler than the one we used in our previous works $[5,28,29]$. That means that the setup performance, tuning, and alignment are much simpler, which excludes a possibility to introduce additional systematic errors into the experimental results.

In [31] the authors demonstrated the first measurement of a so-called "near-field" ODR. In spite of the fact that in their case the detector was placed in the extreme PWZ, it was very difficult to observe the PWZ effect because they used an imaging optical system to measure the ODR spot on the target surface. In their case the PWZ effect must cause the single particle spatial distribution distortion due to the light diffraction on the finite aperture of the optical system. Unfortunately, the authors did not make any estimation on this matter. They used a very simplified model that completely excludes the diffraction phenomenon. A good consistency between the experiment and the theory presented in this paper demonstrates a possibility to perform quantitative estimations for the ODR spatial distribution variations in the experiment presented in [31].

Finally, one should say that the method for PWZ effect suppression is suitable for optical wavelength only. However, the transition and diffraction radiation spectral ranges are very broad. DR in the millimeter and the submillimeter wavelength range has been intensively studied for longitudinal electron beam profile characterization. In this case it is might be possible to use concave (paraboloidal) targets (see, for instance, [19,32]). This method was successfully applied in the experiment [33].

[1] P. Catravas, W. P. Leemans, E. Esarey, M. Zolotorev, D. Whittum, R. Iverson, M. Hogan, and D. Walz, in Proceedings of the 1999 Particle Accelerator Conference, New York, 1999, p. 2111; J. B. Rosenzweig, A. Murokh, and A. Tremaine, AIP Conf. Proc. 472, 38 (1999); G. A. Krafft, J.-C. Denard, R. W. Dickson, and R. Kazimi, LANL, arXiv:physics/0009087. 
[2] R. B. Fiorito, A.G. Shkvarunets, T. Watanabe, V. Yakimenko, and D. Snyder, Phys. Rev. ST Accel. Beams 9, 052802 (2006); R. B. Fiorito, and D. W. Rule, AIP Conf. Proc. 319, 21 (1997), and references therein.

[3] F. Lohl, S. Schreiber, M. Castellano, G. Di Pirro, L. Catani, A. Cianchi, and K. Honkavaara, Phys. Rev. ST Accel. Beams 9, 092802 (2006), and references therein.

[4] C. P. Welsh, E. Bravin, and T. Lefevre, in Proceedings of 2006 European Particle Accelerator Conference, EPAC06, Edinburgh, Scotland, 2006, p. 1220.

[5] P. Karataev, S. Araki, R. Hamatsu, H. Hayano, T. Muto, G. Naumenko, A. Potylitsyn, N. Terunuma, and J. Urakawa, Phys. Rev. Lett. 93, 244802 (2004).

[6] E. Chiadroni, M. Castellano, A. Cianchi, K. Honkavaara, G. Kube, V. Merlo, and F. Stella, in Proceedings of the 2007 Particle Accelerator Conference, PAC'07, Albuquerque, New Mexico, USA, p. 3982, http://www. jacow.org/.

[7] T. Takahashi, Y. Shibata, F. Arai, K. Ishi, T. Ohsaka, M. Ikezawa, Y. Kondo, T. Nakazato, S. Urasawa, R. Kato, S. Niwano, and M. Oyamada, Phys. Rev. E 48, 4674 (1993); Y. Shibata, S. Hasebe, K. Ishi, T. Takahashi, T. Ohsaka, M. Ikezawa, T. Nakazato, M. Oyamada, S. Urasawa, T. Yamakawa, and Y. Kondo, Phys. Rev. E 52, 6787 (1995); Y. Shibata, K. Ishi, T. Takahashi, T. Matsuyama, F. Hinode, and Y. Kondo, Nucl. Instrum. Methods Phys. Res., Sect. A 528, 162 (2004).

[8] O. Grimm, in Ref. [6], p. 2653.

[9] M. Castellano, V. A. Verzilov, L. Catani, A. Cianchi, G. Orlandi, and M. Geitz, Phys. Rev. E 63, 056501 (2001).

[10] H. Lihn, P. Kung, C. Settakorn, H. Wiedemann, D. Bocek, and M. Hernandez, Phys. Rev. Lett. 76, 4163 (1996).

[11] M. Castellano and V. Verzilov, Phys. Rev. ST Accel. Beams 1, 062801 (1998).

[12] M. Castellano, A. Cianchi, G. Orlandi, and V. A. Verzilov, Nucl. Instrum. Methods Phys. Res., Sect. A 435, 297 (1999).

[13] N. F. Shul'ga and S. N. Dobrovol'sky, Phys. Lett. A 259, 291 (1999).

[14] V. A. Verzilov, Phys. Lett. A 273, 135 (2000).

[15] S. N. Dobrovolsky and N.F. Shulga, Nucl. Instrum. Methods Phys. Res., Sect. B 201, 123 (2003), and references therein.

[16] G. A. Naumenko, Nucl. Instrum. Methods Phys. Res., Sect. B 227, 87 (2005).

[17] M.L. Ter-Mikaelyan, High Energy Electromagnetic Processes in Condensed Media (Wiley-Interscience, New York, 1972).
[18] M. Castellano, V. Verzilov, L. Catani, A. Cianchi, G. D’Auria, M. Ferianis, and C. Rossi, Phys. Rev. E 67, 015501(R) (2003).

[19] P. Karataev, Phys. Lett. A 345, 428 (2005).

[20] ATF: Accelerator Test Facility Study Report JFY 19961999, edited by H. Hayano et al. (KEK Internal 2000-6, 2000).

[21] M. Castellano, Nucl. Instrum. Methods Phys. Res., Sect. A 394, 275 (1997).

[22] A. P. Potylitsyn and N. A. Potylitsyna, Izv. Vyssh. Uchebn. Zaved., Fiz. 4, 56 (2000) [Russ. Phys. J. 43, 303 (2000)].

[23] J. Urakawa, H. Hayano, K. Kubo, S. Kuroda, N. Terunuma, M. Kurikia, T. Okugia, N. Naito, S. Araki, A. Potylitsyn, G. Naumenko, P. Karataev, N. Potylitsyna, I. Vnukov, T. Hirose, R. Hamatsu, T. Muto, M. Ikezawa, and Y. Shibata, Nucl. Instrum. Methods Phys. Res., Sect. A 472, 309 (2001).

[24] P. Karataev, S. Araki, R. Hamatsu, H. Hayano, T. Muto, G. Naumenko, A. Potylitsyn, N. Terunuma, and J. Urakawa, Nucl. Instrum. Methods Phys. Res., Sect. B 227, 198 (2005).

[25] R. B. Fiorito and D. W. Rule, Nucl. Instrum. Methods Phys. Res., Sect. B 173, 67 (2001).

[26] Handbook of Optics/Sponsored by the Optical Society of America, edited by Walter G. Driscoll and William Vaughan (McGraw-Hill, New York, 1978).

[27] Handbook of Optical Constants of Solids, edited by Edward D. Palik (Academic Press Orlando, 1985), p. 804.

[28] P. Karataev, S. Araki, R. Hamatsu, H. Hayano, T. Muto, G. Naumenko, A. Potylitsyn, N. Terunuma, and J. Urakawa, Nucl. Instrum. Methods Phys. Res., Sect. B 227, 158 (2005).

[29] P. Karataev, H. Hayano, T. Muto, N. Terunuma, J. Urakawa, R. Hamatsu, G. Naumenko, and A. Potylitsyn, in Proceedings of European Particle Accelerator Conference, EPAC 04, Lucerne, Switzerland, 2004, p. 2646.

[30] SAD, Strategic Accelerator Design, http://acc-physics. kek.jp/SAD/sad.html.

[31] A. H. Lumpkin, W. J. Berg, N. S. Sereno, D. W. Rule, and C.-Y. Yao, Phys. Rev. ST Accel. Beams 10, 022802 (2007).

[32] A. P. Potylitsyn and R. O. Rezaev, Nucl. Instrum. Methods Phys. Res., Sect. B 252, 44 (2006).

[33] B. N. Kalinin, G. A. Naumenko, A. P. Potylitsyn, G. A. Saruev, L. G. Sukhikh, and V. A. Cha, JETP Lett. 84, 110 (2006). 